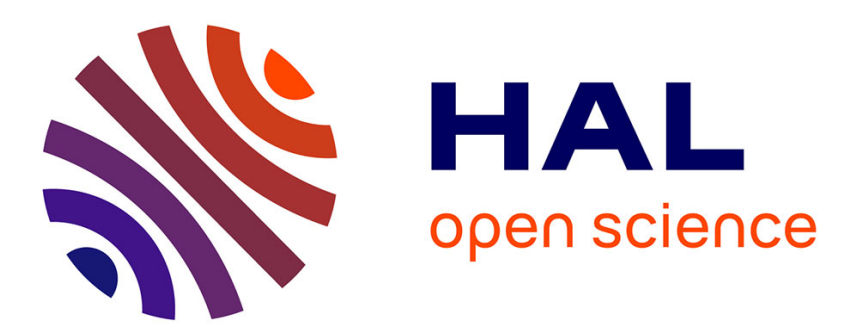

\title{
Infection Time in Multistable Gene Networks. A Backward Stochastic Variational Inequality with Nonconvex Switch-Dependent Reflection Approach
}

\author{
Dan Goreac, Eduard Rotenstein
}

\section{- To cite this version:}

Dan Goreac, Eduard Rotenstein. Infection Time in Multistable Gene Networks. A Backward Stochastic Variational Inequality with Nonconvex Switch-Dependent Reflection Approach. Set-Valued and Variational Analysis, 2016, 10.1007/s11228-016-0382-7 . hal-01211101v2

HAL Id: hal-01211101

$$
\text { https://hal.science/hal-01211101v2 }
$$

Submitted on 4 Sep 2016

HAL is a multi-disciplinary open access archive for the deposit and dissemination of scientific research documents, whether they are published or not. The documents may come from teaching and research institutions in France or abroad, or from public or private research centers.
L'archive ouverte pluridisciplinaire HAL, est destinée au dépôt et à la diffusion de documents scientifiques de niveau recherche, publiés ou non, émanant des établissements d'enseignement et de recherche français ou étrangers, des laboratoires publics ou privés. 


\title{
Infection Time in Multistable Gene Networks. A Backward Stochastic Variational Inequality with Nonconvex Switch-Dependent Reflection Approach
}

\author{
Dan Goreac* $\quad$ Eduard Rotenstein ${ }^{*}$
}

July 27, 2016

\begin{abstract}
We investigate a mathematical model associated to the infection time in multistable gene networks. The mathematical processes are of hybrid switch type. The switch is governed by pure jump modes and linked to DNA bindings. The differential component follows backward stochastic dynamics reflected in some mode-dependent nonconvex domains. First, we study the existence of solutions to the resulting stochastic variational inclusions, by reducing the model to a family of ordinary variational inclusions with generalized reflection in semiconvex domains. Second, by considering control-dependent drivers, we hint to some model-selection approach by embedding the controlled backward stochastic variational inclusion in a family of regular measures. Regularity and structural properties of these sets are given.
\end{abstract}

Keywords : backward stochastic variational inclusion, nonconvex domains, piecewise deterministic Markov processes (PDMP), occupation measures

AMS 2010 subject classification : 60H10, 60G55, 60J75, 92C42, 93E03

\section{Introduction}

The aim of this paper is to study some mathematical properties leading to the detection of infection time in a specific class of stochastic gene networks. The mathematical apparatus is based on a particular class of piecewise deterministic Markov processes (PDMP, first introduced in [21]). The basic example one has in mind is a bistable (multistable) system consisting in a temperate virus and a host. The pathogen is assumed to undergo a lysogenic cycle prior to its release by lysis. We are interested in the detection of the latest infection time of such pathogens. Namely, observing the state of the provirus at some terminal time $T$, we wish to characterize the trajectory (or trajectories) leading to this state. The dynamics are modeled by a piecewise deterministic (controlled) behavior of switch type. The switches are indicated by DNA bindings in specific sites. The lysis region(s) are DNA binding-dependent and are characterized by domains around (stable) critical concentrations. As long as the system is in the lysogenic cycle, these lytic regions are avoided. The randomness is governed by DNA bindings modeled by a pure-jump process. This leads to a problem with switched PDMP dynamics given as a backward stochastic variational

\footnotetext{
*Université Paris-Est, LAMA (UMR 8050), UPEMLV, UPEC, CNRS, F-77454, Marne-la-Vallée, France, Dan.Goreac@u-pem.fr

${ }^{\dagger}$ Acknowledgement. The work of the first author has been partially supported by the French National Research Agency project PIECE, number ANR-12-JS01-0006.

${ }^{\ddagger}$ Faculty of Mathematics, "Alexandru Ioan Cuza" University, Bd. Carol I, no. 9-11, Iasi, Romania

${ }^{\S}$ Acknowledgement. The work of the second author has been partially supported by Grant POSDRU/159/1.5/S/137750, Programe doctorale şi postdoctorale-suport pentru creşterea competitivităţii cercetării in domeniul Ştiinţelor exacte.
} 
inclusion reflected in a nonconvex domain (the exterior of balls, for example). The reflection is assumed to be oblique in the direction of best reachable product concentration and the domains of exclusion may depend on the state of the underlying jump process. For the biological and/or mathematical details of gene networks, the reader is referred to [17], [32], [20], [19], [28].

Our switch process can be described by a couple $(\Gamma, Y$.), where the first component is a purejump process (mode) and, to simplify the framework, it is assumed to take values in some finite set $E$. The mode process is governed by a jump rate $\lambda$ and a transition measure $Q$ depending on the current mode. In the classical, forward formulation, the second component describes a vector of product concentrations and evolves along a mode-dependent flow (say $f$ ). This piecewise deterministic dynamics may also depend on an exogenous control parameter (catalyzer, temperature). In our framework, $Y$ still follows a piecewise-deterministic trajectory, but it is given with respect to its final (random) value at time $T>0$. Moreover, the trajectory is reflected along an oblique direction $H$ such that it remains in a domain dictated by $\Gamma$ (say $\mathcal{O}_{\Gamma}$ ). If one denotes by $q$ the random measure associated to the mode, we deal with a backward stochastic variational inclusion (BSVI, for short) of type

$$
\left\{\begin{array}{cc}
-d Y_{t}^{T, \xi}+H\left(t, Y_{t}^{T, \xi}\right) \partial^{-} \varphi_{\mathcal{O}_{\Gamma_{t-}}}\left(Y_{t}^{T, \xi}\right) d t \ni & \int_{E} f\left(t, \gamma, \Gamma_{t-}, Y_{t-}^{T, \xi}, Z_{t}^{T, \xi}(\gamma), u_{t}\right) \lambda\left(\Gamma_{t-}\right) Q\left(\Gamma_{t-}, d \gamma\right) d t \\
& -\int_{E} Z_{t}^{T, \xi}(\gamma) q(d t d \gamma), \\
Y_{t}^{T, \xi}=\xi . &
\end{array}\right.
$$

The exact definition of solution and the assumptions will be made clear in the following sections.

Backward stochastic differential equations (BSDE, for short) have been introduced in [8] in order to describe the adjoint process in the stochastic version of Pontryagin maximum principle. The concept has been generalized to a Brownian, nonlinear framework by the seminal paper [38]. The authors prove existence and uniqueness for the solution in a Lipschitz setting. The notion has been extended to treat normally reflected Brownian dynamics in [39]. This led to the notion of backward stochastic differential inclusions (BSVI). The method adapts to a backward setting the penalization approach introduced for forward inclusions in [1]. Further developments for a Hilbert setting are given in [40]. Oblique reflection with respect to convex domains arises naturally in the study of optimal switching problems and has been considered in [33], etc. The authors of [25] consider a BSVI governed by Brownian motion and oblique subgradients (in Clarke's sense) on convex domains. They distinguish two cases. In the case of time-dependent oblique direction, they show the existence and uniqueness of a strong solution. Whenever the oblique direction depends also on the incidence point, one produces weak solutions for the equation. The method relies on suitable estimates on the penalized equations. In the obliquely reflected framework on nonconvex domains, the recent paper [41] considers (forward) stochastic variational inequalities using Fréchet-type subdifferentials. In order to tackle the nonconvex setup, the authors extended the approximation method from convex to semiconvex settings. In particular, this allows one to deal with a large class of domains written as the difference of convex sets.

The case of BSDE driven by discontinuous processes has been considered in [5], [43], [44], [13], etc., while the pure-jump case is studied in [14]. In the case of marked point processes, BSDE have been considered in [15]. A different approach relying on iterative solving of ODE (resp. SDE) between consecutive jumps is given in [16] (resp. [35]).

This paper deals with two problems in connection to the theory of backward stochastic variational inclusions for switched PDMP. To our best knowledge, it is the first result on backward stochastic dynamics in which the reflection is given with respect to nonconvex sets. Moreover, our approach allows one to deal with families of sets indexed by the mode and, therefore, having a special structure of time-dependence. First, we give a result on the existence of the solution and the connection with a well-chosen system of ordinary differential reflection problems. This approach 
generalizes to this specific, reflected framework, the recent results of [16]. This reduction has the advantage of allowing to deal with switch-dependent domains and generalized reflection. Second, we envisage an occupation measure embedding approach when the driver $f$ depends on some (predictable) exogenous control. The controlled trajectories corresponding to a given terminal datum are seen as elements of a convenient space of measures. This space is shown to be regular enough (convex, compact) and be given by a convenient inclusion. Support conditions relying these sets to Fréchet subdifferential are also given. This approach can be employed in order to select the parameters best fitting a desired runtime behavior under given terminal restrictions.

The paper is organized as follows. In Section 2 we introduce the motivating example of multistable gene networks. We recall the biological description of Hasty's model [32]. We briefly explain how a switch PDMP is associated to such models and distinguish between the stability domains. We explain why nonconvex (lysogeny) domains naturally appear in these models. Section 3 gathers the results on BSVI. We begin with introducing the construction of the pure-jump mode and the standing assumptions in 3.1. In Subsection 3.2 we study the BSVI with respect to the random measure associated to the mode. We introduce some notations making clear the stochastic structure of several concepts : final data, predictable and càdlàg adapted processes as well as the generator and the compensator of the initial random measure. The notations follow the ordinary differential approach from [16]. The first result (Proposition 7) links the BSVI to a class of iterative ordinary differential inclusions with generalized reflection in semiconvex domains. Next, this system is shown to be solvable in Theorem 10, thus providing the solution of the initial BSVI. Section 4 provides some elements of optimal design. We begin with presenting a case study and emphasizing the main drawbacks for different control parameters in Subsection 4.1. Next, we show how the controlled backward dynamics can be embedded in a suitable space of measures. We begin with embedding the penalized gradient solutions (in Proposition 11) and deduce the properties for the limit set (Theorem 12). Section 5 gathers all the proofs of our assertions.

\section{A Motivating Example}

It is well-known that, in prokaryotes, genes are switched between different states (e.g. on/off) by interactions between specific proteins which intervene at the level of regulation and specific DNA sequences. To better understand the mathematical model we are going to present hereafter, let us concentrate on a basic network presenting bistability of protein concentration and derived from bacteriophage lambda. We consider the repressor expression as described in [32] by the system of biochemical reactions

$$
\left\{\begin{array}{l}
2 X_{1} \stackrel{K_{1}}{\rightleftarrows} X_{2}, D\left(+X_{2}\right) \stackrel{K_{2}}{\rightleftarrows} D X_{2}, D\left(+X_{2}\right) \stackrel{K_{3}}{\rightleftarrows} D X_{2}^{*}, \\
D X_{2}\left(+X_{2}\right) \stackrel{K_{4}}{\rightleftarrows} D X_{2} X_{2}, D X_{2}+P \stackrel{K_{t}}{\longrightarrow} D X_{2}+P+R X_{1}, X_{1} \stackrel{K_{P}}{\rightarrow} .
\end{array}\right.
$$

Biological Description. The authors of [32] propose a genetic applet consisting in a mutant system in which two operator sites (OR2 and OR3) are present. The gene cI expresses repressor (CI), which dimerizes and binds to the DNA as a transcription factor in one of the two available sites. The site OR2 leads to enhanced transcription, while OR3 represses transcription. Using the notations in [32], we let $X_{1}$ stand for the repressor, $X_{2}$ for the dimer, $D$ for the DNA promoter site, $D X_{2}$ for the binding to the OR2 site, $D X_{2}^{*}$ for the binding to the OR3 site and $D X_{2} X_{2}$ for the binding to both sites. We also denote by $P$ the RNA polymerase concentration and by $R$ the number of proteins per mRNA transcript. Therefore, the system consists in a "four" tandem DNA sites and the recognition of lytic/lysogenic patterns follows by identifying the regulatory repressor cI (here described by its $X_{1}$ concentration). The phage can switch between two states : lysogenic (when the repressor is synthesized), respectively lytic which is initiated by DNA damage leading to transcription of cI being turned off. The capital letters $K_{i}, 1 \leq i \leq 4$ for the reversible reactions 
correspond to couples of direct/reverse speed functions $k_{i}, k_{-i}$, while $K_{t}$ and $K_{d}$ only to direct speed functions $k_{t}$ and $k_{d}$.

Mathematical Model. Our simplifying mathematical approach considers a two-scale model (see, for instance [19]). We assume the binding operations to be given by a priori statistical estimates which lead to a Markov pure-jump process $\Gamma$ describing

$$
D \stackrel{K_{2}}{\rightleftarrows} D X_{2}, D \stackrel{K_{3}}{\rightleftarrows} D X_{2}^{*}, D X_{2} \stackrel{K_{4}}{\rightleftarrows} D X_{2} X_{2} \text {. }
$$

The remaining equations will give the continuous flow.

The Jump. The state space of this component is obviously discrete consisting of standard vectors basis of $\mathbb{R}^{4}\left(E=\left\{e_{1}, e_{2}, e_{3}, e_{4}\right\}^{1}\right)$. Switching between these states is given at random times generated according to the propensity function computed starting from the current state (e.g. [26]). For example, at state $e_{1}$ (corresponding to unoccupied DNA $D$ ), the possible reactions are given by

$$
D \stackrel{k_{2}}{\rightarrow} D X_{2}, D \stackrel{k_{3}}{\rightarrow} D X_{2}^{*},
$$

which leads to a total propensity $\lambda\left(e_{1}\right):=k_{2}+k_{3}$ and the postjump position is given, again as in $[26]$, by

$$
Q\left(e_{1}, d e\right)=\frac{k_{2}}{\lambda\left(e_{1}\right)} \delta_{e_{2}}(d e)+\frac{k_{3}}{\lambda\left(e_{1}\right)} \delta_{e_{3}}(d e) .
$$

Here, $\delta$ stands for the standard Dirac mass. Similar assertions hold true for the remaining reactions.

The Continuous Flow. While in lysogenic state (note that the dimer intervenes at binding level), repressor and dimer concentrations are given by an ordinary differential equation (ODE)

$$
\left\{\begin{array}{l}
\frac{d X_{1}}{d t}=f_{1}\left(\Gamma_{t}, X\right):=-2 k_{1} X_{1}^{2}-k_{d} X_{1}+2 k_{-1} X_{2}+R 1_{\Gamma_{t}=e_{2},|X| \text { large enough }} \\
\frac{d X_{2}}{d t}=f_{2}\left(\Gamma_{t}, X\right):=k_{1} X_{1}^{2}-k_{-1} X_{2}
\end{array}\right.
$$

In lytic state, transcription of cI repressor is turned off.

Lysogenic Domain(s). In an attempt to distinguish between symbiotic (lysogenic) behavior and the excision of the virus, it is, therefore, natural to set the lysogenic domain $\mathcal{O}$ to be the exterior of some regions "around $(0,0)$ " (or other stable points). A careful look at our model shows that the need of dimer is not the same at all DNA states. Indeed, while in state $e_{2}$ (occupation of the promoter site), the system is equally likely to free the dimer (in $D\left(+X_{2}\right) \stackrel{k_{-1}}{\leftarrow} D X_{2}$ ) or to consume it (in $\left.D X_{2}\left(+X_{2}\right) \stackrel{k_{4}}{\Rightarrow} D X_{2} X_{2}\right)$. Then, we get a lysogenic domain $\mathcal{O}_{2}=\left\{\left(x_{1}, x_{2}\right) \in\left[0, \rho_{\max }\right]: x_{1}^{2}+x_{2}^{2} \geq r\right\}$ such as in Fig 1.1. However, as the state is free DNA $\left(e_{1}\right)$, all reactions need dimer binding. It is then natural to consider a domain of type $\mathcal{O}_{1}=\left\{\left(x_{1}, x_{2}\right) \in\left[0, \rho_{\max }\right]: x_{1}^{2}+\frac{1}{4} x_{2}^{2} \geq r\right\}$ (see Fig. 1.2).

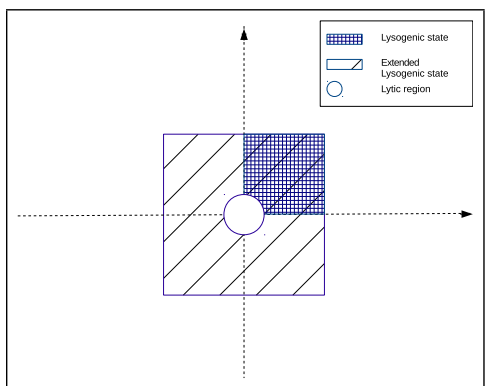

Fig. 1.1 Lysogenic/Lytic Domains for $e_{2}$

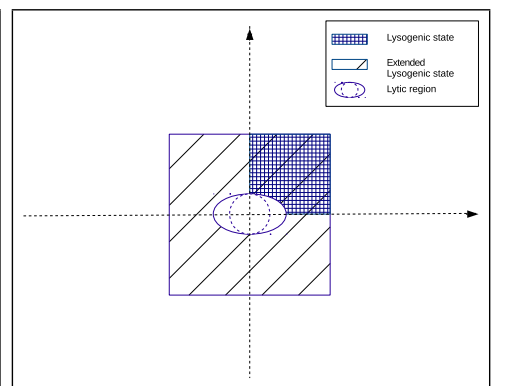

Fig. 1.2 Lysogenic/Lytic

Domains for $e_{1}$

\footnotetext{
${ }^{1}$ In Section 4, in order to avoid confusion with the trajectories of the marked point process, the elements of $E$ will simply be given by their index 1,2,3,4. The lysogeny sets $\mathcal{O}$ can also have $e_{i}$ or $i$ as an index.
} 
Similar constructions are possible for $\mathcal{O}_{3}$ and $\mathcal{O}_{4}$. To simplify arguments, we assume hereafter the simplest form i.e. $\mathcal{O}_{3}=\mathcal{O}_{4}=\mathcal{O}_{2}$.

Reverse Engineering : Backward Dynamics and Reflection Directions. The reversible biochemical equations allows to "guess" the constants by the so-called "law of mass action" going back to the considerations of [31]. These hints are valid at equilibrium when the state is invariant. The actual values of these constants are usually taken from different tables available in the literature and using various normalizations (see, for example, [20]). As consequence, even in the simplest model, different parameters lead to different behaviors (slow unstable, fast unstable, stable, etc. ). Instead of switching between different parameters and finding those that "fit" a targeted behavior, we propose a reverse validation procedure as follows : based on the observation of the system at some time $T$ (given in an absolute time framework), one solves the backward equation with fixed parameters until the process passes too much time on the frontier of the lysogenic domain. At this time, it is reasonable to think that the symbiotic model is no longer valid (the trajectory is kept within the lysogenic domain only because of the reflection penalty) and another model (independent of the infection) should be considered. It is, therefore, the time at which the independent bacterium becomes a host for the lambda virus (infection time). As a by-product, in continuous switching model (see [20] for examples), the presence of a non-zero predictable projection implies that the parameters are not fitted.

Assume, for the time being that, at some time $T>0$, the phage lambda has been functioning on a lysogenic pathway starting at some time $t_{0}$. Then, the trajectory has been reflected such that to remain in the lysogenic domains $\mathcal{O}_{i}$. While many type of reflection can be considered, we will assume here that the virus is driven by the best reachable stable state as follows. If the current DNA state is 3 , the only reachable DNA state is 1 . In this case, the only stable point of the ODE (for both the current state $\gamma=3$ and reachable state $\gamma=1$ ) is $(0,0)$ and we will consider normal reflection to $\mathcal{O}_{3}$. Similar type of reflection is considered for $\mathcal{O}_{4}$. If the current DNA state is 2 , then there exists another critical point $\left(\frac{R}{k_{d}}, \frac{k_{1} R^{2}}{k_{-1} k_{d}^{2}}\right)$ and the reflection is done using as axis the line joining this critical point to the incidence point (see Fig 2.). If the DNA state is set to 1, binding to the promoter site is envisaged and the reflection is done as before.

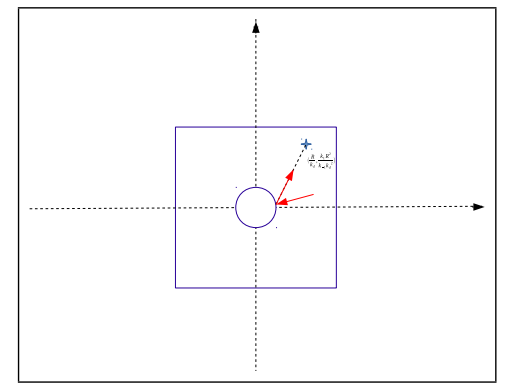

Fig 2. Oblique reflection

along best reachable state

Reverse-engeneering from time $T$ in order to detect the time of infection leads to the attempt of solving a backward differential equation adapted to the underlying DNA (Markov) mechanism and reflected in the nonconvex domains $\mathcal{O}_{i}$. We emphasize that, in our framework, the domains are allowed to vary in time (at time $t$ they depend on the mode $\Gamma_{t-}$ ).

Control. In our phage model, the hybrid mechanism is essentially governed by the reaction speeds $k$. Nevertheless, these speeds can be altered in various ways. To give a simple example, the reaction constants in [17] vary in simulations with the choice of the product half time denoted $T p\left(1 \mathrm{~h}, 2 \mathrm{~h}, 4 \mathrm{~h}\right.$, etc.) and the stability of the regime (e.g. $\frac{4 \ln 2}{T p}$ for slow-unstable, $\frac{40 \ln 2}{T p}$ for fastunstable, etc.). Therefore, designing the best model amounts to considering that our differential 
mechanism is governed by an exogenous control parameter that can be associated to temperature and/or catalysts conditions. Then, the construction of the differential component is given by a driver depending on a predictable control process $u$.

\section{The Backward Stochastic Variational Inclusion}

\subsection{Preliminaries and Technical Assumption}

\subsubsection{Markov Jump Processes}

We briefly recall the construction of a particular class of Markov pure jump, non explosive processes on a space $\Omega$ and taking their values in a metric space $(E, \mathcal{B}(E))$. For the explicit construction of $\Omega$ (using the Hilbert cube), the reader is referred to [22, Section 23]. Here, $\mathcal{B}(E)$ denotes the Borel $\sigma$-field of $E$. The elements of the space $E$ are referred to as modes. These elements can be found in [22] in the particular case of piecewise deterministic Markov processes; see also [10]. In all generality, $E \subset \mathbb{R}^{m^{\prime}}$, for some $m^{\prime} \geq 1$. The process is completely described by a couple $(\lambda, Q)$ constituting on:

(i) a Lipschitz continuous jump rate $\lambda: E \longrightarrow \mathbb{R}_{+}$such that $\sup _{\theta \in E}|\lambda(\theta)| \leq c_{0}$ and

(ii) a transition measure $Q: E \longrightarrow \mathcal{P}(E)$, where $\mathcal{P}(E)$ stands for the set of probability measures on $(E, \mathcal{B}(E))$ such that:

$$
\left\{\begin{aligned}
&\left(i i_{1}\right) \quad Q(\gamma,\{\gamma\})=0 ; \\
&\left(i i_{2}\right) \quad \text { for each bounded, uniformly continuous } h \text {, there exists a continuous } \eta_{h}: \mathbb{R} \longrightarrow \mathbb{R}_{+}, \\
& \text {such that } \eta_{h}(0)=0 \text { and }\left|\int_{E} h(\theta) Q(\gamma, d \theta)-\int_{E} h(\theta) Q\left(\gamma^{\prime}, d \theta\right)\right| \leq \eta_{h}\left(\left|\gamma-\gamma^{\prime}\right|\right) . \\
&\text { (The distance } \left.\left|\gamma-\gamma^{\prime}\right| \text { is the usual Euclidian one on } \mathbb{R}^{m} .\right)
\end{aligned}\right.
$$

Given an initial mode $\gamma_{0} \in E$, the first jump time has a conditional law $\mathbb{P}^{\gamma_{0}}\left(T_{1} \geq t\right)=\exp \left(-t \lambda\left(\gamma_{0}\right)\right)$. The process $\Gamma_{t}:=\gamma_{0}$, on $t<T_{1}$. The post-jump location $\gamma_{1}$ has $Q\left(\gamma_{0}, \cdot\right)$ as conditional distribution. Next, we select the inter-jump time $T_{2}-T_{1}$ such that $\mathbb{P}^{\gamma_{0}}\left(T_{2}-T_{1} \geq t / T_{1}, \gamma_{1}\right)=\exp \left(-t \lambda\left(\gamma_{1}\right)\right)$ and let us set $\Gamma_{t}:=\gamma_{1}$, if $t \in\left[T_{1}, T_{2}\right)$. The post-jump location $\gamma_{2}$ satisfies $\mathbb{P}^{\gamma_{0}}\left(\gamma_{2} \in A / T_{2}, T_{1}, \gamma_{1}\right)=$ $Q\left(\gamma_{1}, A\right)$, for all Borel set $A \subset E$. And so on. Similar construction can be given for a non-zero initial starting time (i.e. a pair $\left.\left(t, \gamma_{0}\right)\right)$.

We look at the process $\Gamma$ under $\mathbb{P}^{\gamma_{0}}$ and denote by $\mathbb{F}^{0}$ the filtration $\left(\mathcal{F}_{[0, t]}:=\sigma\left\{\Gamma_{r}: r \in[0, t]\right\}\right)_{t \geq 0}$. The predictable $\sigma$-algebra will be denoted by $\mathcal{P}^{0}$ and the progressive $\sigma$-algebra by $\operatorname{Prog}^{0}$. As usual, we introduce the random measure $q$ on $\Omega \times[0, \infty] \times E$ by setting

$$
q(\omega, A)=\sum_{k \geq 1} 1_{\left(T_{k}(\omega), \Gamma_{T_{k}(\omega)}(\omega)\right) \in A} \text {, for all } \omega \in \Omega, A \in \mathcal{B}([0, \infty]) \times \mathcal{B}(E) .
$$

The compensator of $q$ is $\widehat{q}(d s), d \theta:=\lambda\left(\Gamma_{s-}\right) Q\left(\Gamma_{s-}, d \theta\right) d s$ and the compensated martingale measure is given by

$$
\widetilde{q}(d s d \theta):=q(d s d \theta)-\lambda\left(\Gamma_{s-}\right) Q\left(\Gamma_{s-}, d \theta\right) d s .
$$

Following the general theory of integration with respect to random measures (see, for example [34]), we denote by $\mathcal{L}^{r}\left(q ; \mathbb{R}^{N}\right)$ the space of all $\mathcal{P}^{0} \otimes \mathcal{B}(E)$ - measurable, $\mathbb{R}^{N}$-valued functions $H_{s}(\omega, \theta)$ on $\Omega \times \mathbb{R}_{+} \times E$ such that

$\mathbb{E}^{\gamma_{0}}\left[\int_{0}^{T} \int_{E}\left|H_{s}(\theta)\right|^{r} q(d s d \theta)\right]=\mathbb{E}^{\gamma_{0}}\left[\int_{0}^{T} \int_{E}\left|H_{s}(\theta)\right|^{r} \lambda\left(\Gamma_{s-}\right) Q\left(\Gamma_{s-}, d \theta\right) d s\right]<\infty$, for all $T<\infty$.

Here, $N \in \mathbb{N}^{*}$ and $r \geq 1$ is a real parameter. By abuse of notation, whenever no confusion is at risk, the family of processes satisfying the above condition for a fixed $T>0$ will still be denoted by $\mathcal{L}^{r}\left(q ; \mathbb{R}^{M}\right)$. 
To keep arguments simple, we will be dealing with a finite set of modes $E=\{1,2, \ldots, p\}$, for some $p \geq 2$. Moreover, at some point we will assume that the observations on the DNA are only made up to time $T_{M}$ for some $M \in \mathbb{N}^{*}$. Then, we will need to modify $q$ to take into account this condition as well as a terminal time $T>0$ (see Subsection 3.2.1).

\subsubsection{Technical Assumptions}

We begin with recalling some useful notions (semiconvexity, Fréchet subdifferential) and the link between these concepts. We also give the standing assumptions on our driver.

Definition 1 Given a non-negative real constant $\beta \geq 0$, the non-empty set $\mathcal{O} \subset \mathbb{R}^{m}$ is called $\beta$ semiconvex if, for every $x \in B d(\mathcal{O})$, there exists $\hat{x} \in \mathbb{R}^{m} \backslash\{0\}$ such that $\langle\hat{x}, y-x\rangle \leq \beta|\hat{x}||y-x|^{2}$, for all $y \in \mathcal{O}$. (Here $B d(\mathcal{O})$ denotes the boundary of $\mathcal{O}$.)

Remark $2 A$ non-empty, closed subset $\mathcal{O} \subset \mathbb{R}^{m}$ is $\beta$-semiconvex if and only if it satisfies, the so-called "uniform exterior ball condition" (for short, $1 / 2 \beta$-UEBC) i.e. if, for every $x \in B d(\mathcal{O})$, the normal (exterior) cone $\mathcal{N}_{\mathcal{O}}(x) \neq\{0\}$ and, for every $u \in \mathcal{N}_{\mathcal{O}}(x)$ for which $|u|=1 / 2 \beta$, one has $d_{\mathcal{O}}(x+u)=1 / 2 \beta$. As usual, $d_{\mathcal{O}}$ denotes the distance function to the set $\mathcal{O}$. For further details on the subject, the reader is referred to [41].

For a given closed set $\mathcal{O} \subset \mathbb{R}^{m}$ and for $\varepsilon>0$, we denote by

$$
\begin{aligned}
& \mathbb{B}(\mathcal{O}, \varepsilon):=\left\{x \in \mathbb{R}^{m}: d_{\mathcal{O}}(x)<\varepsilon\right\} \text { its (open) } \varepsilon \text {-neighborhood and } \\
& \overline{\mathbb{B}}(\mathcal{O}, \varepsilon):=\left\{x \in \mathbb{R}^{m}: d_{\mathcal{O}}(x) \leq \varepsilon\right\} \text { its (closed) } \varepsilon \text {-neighborhood. }
\end{aligned}
$$

Let us consider a function $\varphi_{\mathcal{O}}: \mathbb{R}^{m} \rightarrow(-\infty,+\infty]$ such that $\operatorname{Dom}\left(\varphi_{\mathcal{O}}\right):=\left\{y \in \mathbb{R}^{m}: \varphi(y)<+\infty\right\}$. For the function $\varphi_{\mathcal{O}}$ we assume that $\operatorname{Dom}\left(\varphi_{\mathcal{O}}\right)=\mathcal{O}$. We recall that the Fréchet subdifferential of $\varphi_{\mathcal{O}}$ at $x \in \mathbb{R}^{m}$ is given by

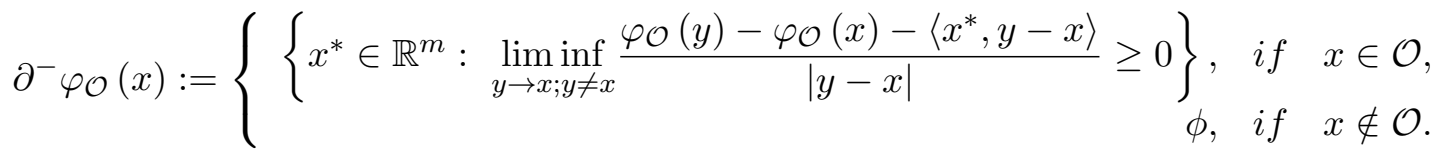

As before, we let $\operatorname{Dom}\left(\partial^{-} \varphi_{\mathcal{O}}\right):=\left\{x \in \mathbb{R}^{m}: \partial^{-} \varphi_{\mathcal{O}}(x) \neq \emptyset\right\}$.

Remark 3 In the particular case of the convexity indicator function of some closed set $\mathcal{O}$ (i.e. $\varphi(x):=I_{\mathcal{O}}(x)=0$, if $x \in \mathcal{O}$ and $+\infty$, otherwise), $\varphi$ is a lower semicontinuous function and its subdifferential operator is $\partial^{-} I_{\mathcal{O}}(x)=\mathcal{N}_{\mathcal{O}}(x)$, for all $x \in \mathcal{O}$.

The reader is invited to note that the domains appearing in our example (cf. Fig. 1) are not convex. Nevertheless, they enjoy some smoothness properties as follows.

Definition 4 Given two non-negative real constants $\rho, \beta \geq 0$ a function $\varphi: \mathbb{R}^{m} \rightarrow(-\infty,+\infty]$ is called $(\rho, \beta)$-semiconvex if

(i) $\overline{\operatorname{int}(\operatorname{Dom}(\varphi))}=\overline{\operatorname{Dom}(\varphi)}$ is $\beta$-semiconvex,

(ii) $\operatorname{Dom}\left(\partial^{-} \varphi\right) \neq \emptyset$ and, for every $\left(x, x^{*}\right) \in \partial^{-} \varphi$ and $y \in \mathbb{R}^{m}$, one has

$$
\left\langle x^{*}, y-x\right\rangle+\varphi(x) \leq \varphi(y)+\left(\rho+\beta\left|x^{*}\right|\right)|y-x|^{2} .
$$

Given two non-negative real constants $\rho, \beta \geq 0$, we consider a family of mode-indexed, $(\rho, \beta)$ semiconvex functions $\varphi_{\mathcal{O}_{\gamma}}: \mathbb{R}^{m} \rightarrow(-\infty,+\infty]$ and assume

$$
\overline{\operatorname{Dom}\left(\varphi_{\mathcal{O}_{\gamma}}\right)}=\mathcal{O}_{\gamma} \text { is bounded, }
$$


for all $\gamma \in E$. The oblique direction will be given by a continuous symmetric matrix-valued function $H: \mathbb{R}_{+} \times \mathbb{R}^{m} \longrightarrow \mathcal{S}_{m}^{+}$satisfying

$$
\left\{\begin{array}{l}
\text { i) } \quad\left|H(t, y)-H\left(t, y^{\prime}\right)\right|+\left|(H(t, y))^{-1}-\left(H\left(t, y^{\prime}\right)\right)^{-1}\right| \leq c_{H}\left|y-y^{\prime}\right| \\
\text { ii) } \quad \frac{1}{c_{H}}|u|^{2} \leq\langle H(t, y) u, u\rangle \leq c_{H}|u|^{2} \text {, for all } u \in \mathbb{R}^{m}
\end{array}\right.
$$

for some $c_{H}>0$ and all $t \in \mathbb{R}_{+},\left(y, y^{\prime}\right) \in \mathbb{R}^{2 m}$. Here, $\mathcal{S}_{m}^{+}$stands for the family of symmetric, positive-definite real-valued matrix of $m \times m$ type. One easily checks that the inverse of $H$ equally satisfies $\left(\mathrm{A}_{\left.H^{-}-i i\right)}\right.$.

We consider that the driver function $f: \mathbb{R}_{+} \times E \times E \times \mathbb{R}^{m} \times \mathbb{R}^{m} \longrightarrow \mathbb{R}^{m}$ is globally continuous, bounded and there exists some constant $c_{f}>0$ such that

$$
\left|f\left(t, \gamma, \gamma^{\prime}, y, z\right)-f\left(t, \gamma, \gamma^{\prime}, y^{\prime}, z^{\prime}\right)\right| \leq c_{f}\left(\left|y-y^{\prime}\right|+\left|z-z^{\prime}\right|\right)
$$

for all $\left(t, \gamma, \gamma^{\prime}, y,, y^{\prime}, z, z^{\prime}\right) \in \mathbb{R}_{+} \times E \times E \times \mathbb{R}^{4 m}$.

Remark 5 The method can be applied to more general drivers (e.g. random, linear growth). However, since all the domain of interest are bounded and we are primarily interested in drivers associated to our biological model (without $z$ component), we have chosen to limit ourselves to this assumption.

\subsection{The Main Results on BSVI}

In connection to our model, for some fixed terminal time $T>0$, we consider the following backward stochastic variational inclusion with mode-dependent reflection :

$$
\left\{\begin{array}{cc}
-d Y_{t}^{T, \xi}+H\left(t, Y_{t}^{T, \xi}\right) \partial^{-} \varphi_{\mathcal{O}_{\Gamma_{t-}}}\left(Y_{t}^{T, \xi}\right) d t \ni \int_{E} f\left(t, \gamma, \Gamma_{t-}, Y_{t-}^{T, \xi}, Z_{t}^{T, \xi}(\gamma)\right) \widehat{q}(d t, d \gamma) \\
-\int_{E} Z_{t}^{T, \xi}(\gamma) q(d t d \gamma) \\
Y_{T}^{T, \xi}=\xi \in \mathbb{L}^{0}\left(\Omega, \mathcal{F}_{T}, \mathbb{P}^{\gamma_{0}} ; \mathbb{R}^{m}\right),
\end{array}\right.
$$

$\mathbb{P}^{\gamma_{0}}$-almost everywhere. We consider an additional cemetery state $\Delta \in \mathbb{R}^{m}$ acting as an indicator of the infection time. As we will see afterwards, this equation can be linked to a system of reflected ordinary differential equations. With this in mind, the coherence of our solution will have to be ensured at jumping times. In other words, one would need the solution $Y_{t}^{T, \xi}$ to belong to $\mathcal{O}_{\Gamma_{t-}}$ and will check this condition at switching times. Should this condition fail to hold, the trajectory will be sent to $\Delta$ (lysogenic pathway is not coherent with the model prior to this time) and remains at $\Delta$ for any time before.

The definition of a solution is given, as usual, by a triplet $\left(Y^{T, \xi}, Z^{T, \xi}, K^{T, \xi}\right)$ in which the latter components take into account the adaptness, respectively a feedback correction for $Y^{T, \xi}$. 
Definition 6 A solution of (1) consists of a triplet $\left(Y_{t}^{T, \xi}, Z_{t}^{T, \xi}, K_{t}^{T, \xi}\right)$ such that:

(i) 1. The process $Y^{T, \xi}$ is càdlàg and continuous except, maybe, at switching times.

2. For $\mathbb{P}^{\gamma_{0}} \times$ Leb -almost all $(\omega, t)$ such that $T_{n}(\omega) \leq t<T_{n+1}(\omega), Y_{t}^{T, \xi} \in \mathcal{O}_{\Gamma_{T_{n}}}$.

3. If $Y_{T_{n}}^{T, \xi}(\omega) \notin \mathcal{O}_{\Gamma_{T_{n-1}}}$, then $Y_{s}^{T, \xi}(\omega)=\Delta$, for almost all $s<T_{n}(\omega)$.

(ii) 1. The process $Z^{T, \xi}(\cdot)$ is $\mathbb{R}^{m}$-valued, $\mathbb{F}$-predictable and

2. $\mathbb{E}^{\gamma_{0}}\left[\int_{0}^{T} \int_{E}\left|Z_{t}^{T, \xi}(\gamma)\right| \widehat{q}(d t, d \gamma)\right]<\infty$.

(iii) 1. The process $K^{T, \xi}$ is $\mathbb{F}$-adapted and $\int_{0}^{T}\left|K_{t}^{T, \xi}\right|^{2} d t<\infty, \mathbb{P}^{\gamma_{0}}$-almost everywhere.

2. For $\mathbb{P}^{\gamma_{0}} \times$ Leb-almost all $(\omega, t)$ such that $T_{n}(\omega) \leq t<T_{n+1}(\omega)$, one has

$$
\left(K_{t}^{T, \xi}(\omega), Y_{t}^{T, \xi}(\omega)\right) \in\left(\partial^{-} \varphi_{\mathcal{O}_{\Gamma_{T_{n}(\omega)}(\omega)}}\left(Y_{t}^{T, \xi}(\omega)\right) \times \mathbb{R}^{m}\right) \cup\{(0, \Delta)\} .
$$

(iv) One has, $\mathbb{P}^{\gamma_{0}} \times$ Leb -almost everywhere,

$$
\begin{gathered}
Y_{t}^{T, \xi}+\int_{t}^{T} H\left(Y_{s}^{T, \xi}\right) K_{s}^{T, \xi} d s+\sum_{n \geq 0, t<T_{n} \leq T} Z_{T_{n}}^{T, \xi}\left(\Gamma_{T_{n}}\right) \\
=\xi+\int_{t}^{T} \int_{E} f\left(s, \gamma, Y_{s}^{T, \xi}, Z_{s}^{T, \xi}\right) \lambda\left(\Gamma_{s}\right) Q\left(\Gamma_{s}, d \gamma\right) d s .
\end{gathered}
$$

Moreover, unless stated otherwise, we will assume that the mode process jumps at most $M>0$ times prior to $T>0$, i.e.

$$
\mathbb{P}^{\gamma_{0}}\left(T_{M+1}=\infty\right)=1 .
$$

This assumption is not a heavy restriction. Indeed, passing to an infinite number of jumps is got by a localization procedure (see [16, Proof of Theorem 3]) and using the special form of our random measure. For this reason, we prefer to concentrate on the specificity of our reflection setting.

\subsubsection{Measurability Issues, Driver and Compensator}

Before giving the reduction of our equation to a system of ODE, we need to introduce some notations making clear the stochastic structure of several concepts : final data, predictable and càdlàg adapted processes as well as the driver and the compensator of the initial random measure. The notations in this subsection follow the ordinary differential approach from [16]. Since we are only interested in what happens on $[0, T]$, we introduce a cemetery state $(\infty, \bar{\gamma})$ which will incorporate all the information after $T \wedge T_{M}$. It is clear that the conditional law of $T_{n+1}$ given $\left(T_{n}, \Gamma_{T_{n}}\right)$ is now composed by an exponential part on $\left[T_{n} \wedge T, T\right]$ and an atom at $\infty$. Similarly, the conditional law of $\Gamma_{T_{n+1}}$ given $\left(T_{n+1}, T_{n}, \Gamma_{T_{n}}\right)$ is the Dirac mass at $\bar{\gamma}$ if $T_{n+1}=\infty$ and given by $Q$ otherwise. Finally, under the assumption $\mathrm{A}_{M}$, after $T_{M}$, the marked point process is concentrated at the cemetery state.

We set $\bar{E}_{T}:=([0, T] \times E) \cup\{(\infty, \bar{\gamma})\}$. For every $n \geq 1$, we let $\bar{E}_{T, n} \subset\left(\bar{E}_{T}\right)^{n+1}$ be the set of all marks of type $e=\left(\left(t_{0}, \gamma_{0}\right), \ldots,\left(t_{n}, \gamma_{n}\right)\right)$ where

$$
\left\{\begin{array}{l}
t_{0}=0, \quad\left(t_{i}\right)_{0 \leq i \leq n} \text { is non-decreasing; } \\
\text { for every } 0 \leq i \leq n-1, \text { if } t_{i} \leq T, \text { then } t_{i}<t_{i+1} ; \\
\text { for every } 0 \leq i \leq n-1, \text { if } t_{i}>T, \text { then }\left(t_{i}, \gamma_{i}\right)=(\infty, \bar{\gamma}),
\end{array}\right.
$$

and endow it with the family of all Borel sets $\mathcal{B}_{n}$. For these sequences, the maximal time is denoted by $|e|:=t_{n}$. Moreover, by abuse of notation, we set $\gamma_{|e|}:=\gamma_{n}$. Whenever $T \geq t>|e|$, we set

$$
e \oplus(t, \gamma):=\left(\left(t_{0}, \gamma_{0}\right), \ldots,\left(t_{n}, \gamma_{n}\right),(t, \gamma)\right) \in \bar{E}_{T, n+1} .
$$


By defining

$$
e_{n}:=\left(\left(0, \gamma_{0}\right),\left(T_{1}, \Gamma_{T_{1}}\right), \ldots,\left(T_{n}, \Gamma_{T_{n}}\right)\right),
$$

we get an $\bar{E}_{T, n}$-valued random variable, corresponding to our mode trajectories.

Let us now express the different notions (final condition, adapted process, predictable process, etc.) with respect to this framework.

The final data $\xi$ is $\mathcal{F}_{T}$-measurable and, thus, for every $n \geq 0$, there exists a $\mathcal{B}_{n} / \mathcal{B}\left(\mathbb{R}^{m}\right)$-measurable function $\bar{E}_{T, n} \ni e \mapsto \xi^{n}(e) \in \mathbb{R}^{m}$ such that:

$$
\left\{\begin{array}{l}
\text { If }|e|=\infty \text {, then } \xi^{n}(e)=0 . \\
\text { Otherwise, on } T_{n}(\omega) \leq T<T_{n+1}(\omega), \xi(\omega)=\xi^{n}\left(e_{n}(\omega)\right) .
\end{array}\right.
$$

A càdlàg process $Y$ continuous except, maybe, at switching times $T_{n}$ is given by the existence of a family of $\mathcal{B}_{n} \otimes \mathcal{B}([0, T]) / \mathcal{B}\left(\mathbb{R}^{m}\right)$-measurable functions $y^{n}$ such that:

$$
\left\{\begin{array}{l}
\text { For all } e \in \bar{E}_{T, n}, y^{n}(e, \cdot) \text { is continuous on }[0, T] \text { and constant }[0, T \wedge|e|] . \\
\text { If }|e|=\infty \text {, then } y^{n}(e, \cdot)=0 . \\
\text { Otherwise, on } T_{n}(\omega) \leq t<T_{n+1}(\omega), y_{t}(\omega)=y^{n}\left(e_{n}(\omega), t\right) \text {, for all } t \leq T .
\end{array}\right.
$$

Similar, an $\mathbb{R}^{m}$-valued $\mathbb{F}$-predictable process $Z$ defined on $\Omega \times[0, T] \times E$ is given by the existence of a family of $\mathcal{B}_{n} \otimes \mathcal{B}([0, T]) \otimes \mathcal{B}(E) / \mathcal{B}\left(\mathbb{R}^{m}\right)$-measurable functions $z^{n}$ satisfying

$\left\{\right.$ If $|e|=\infty$, then $z^{n}(e, \cdot, \cdot)=0$.

Otherwise, on $T_{n}(\omega)<t \leq T_{n+1}(\omega), z_{t}(\omega, \gamma)=z^{n}\left(e_{n}(\omega), t, \gamma\right)$, for all $t \leq T$ and $\gamma \in E$.

To deduce the form of the compensator, one takes into account $\left(\mathrm{A}_{M}\right)$ and simply writes:

(8)

$$
\left\{\begin{array}{l}
\text { If } n \leq M-1, \\
\widehat{q}_{e}^{n}(d t, d \gamma):=\lambda\left(\gamma_{|e|}\right) Q\left(\gamma_{|e|}, d \gamma\right) 1_{|e|<\infty, t \in[|e|, T]} \operatorname{Leb}(d t)+\delta_{\bar{\gamma}}(d \gamma) \delta_{\infty}(d t) 1_{(|e|<\infty, t>T) \cup|e|=\infty}, \\
\text { If } n \geq M, \text { then } \widehat{q}_{e}^{n}(d t, d \gamma)=\delta_{\bar{\gamma}}(d \gamma) \delta_{\infty}(d t) \\
\widehat{q}(\omega, d t, d \gamma):=\sum_{n=0} \widehat{q}_{e_{n}(\omega)}^{n}(d t, d \gamma) 1_{T_{n}(\omega)<t \leq T_{n+1}(\omega) \wedge T} .
\end{array} .\right.
$$

Finally, given a predictable process $z:=\left(z^{n}\right)$, the driver is given by a family of $\mathcal{B}_{n} \otimes \mathcal{B}([0, T]) \otimes$ $\mathcal{B}(E) \otimes \mathcal{B}\left(\mathbb{R}^{m}\right) \otimes \mathcal{B}\left(\mathbb{R}^{m}\right) / \mathcal{B}\left(\mathbb{R}^{m}\right)$-measurable functions

$$
f\left(z^{n}\right)^{n}: \bar{E}_{T, n} \times[0, T] \times E \times \mathbb{R}^{2 m} \longrightarrow \mathbb{R}^{m}
$$

such that:

$$
\left\{\begin{array}{l}
\text { If }|e|<\infty \text { and } n \leq M-1, \text { then, for all }\left(e, t, \gamma, y, y^{\prime}, w, w^{\prime}\right) \in \bar{E}_{T, n} \times[0, T] \times E \times \mathbb{R}^{4 m} \\
\left|f\left(z^{n}\right)^{n}(e, t, \gamma, y, w)-f\left(z^{\prime}, n\right)^{n}\left(e, t, \gamma, y^{\prime}, w^{\prime}\right)\right| \\
\quad \leq c\left(\left|y-y^{\prime}\right|+\left|w-w^{\prime}\right|+\sum_{\gamma^{\prime} \in E}\left|z^{n}\left(e, t, \gamma^{\prime}\right)-z^{\prime n}\left(e, t, \gamma^{\prime}\right)\right| Q\left(\gamma_{|e|}, d \gamma^{\prime}\right)\right) \\
\text { Otherwise, } f\left(z^{n}\right)^{n}(e, \cdot, \cdot, \cdot, \cdot)=0
\end{array}\right.
$$

In this case, we identify the driver as follows.

(10)

Whenever $T_{n}<t \leq T_{n+1}$, we have $f\left(t, \gamma, \Gamma_{t-}, y, \zeta\right)=f\left(z^{n}\right)^{n}\left(e_{n}(\omega), t, \gamma, y, \zeta(\gamma)-z^{n}\left(e_{n}(\omega), t, \gamma\right)\right)$.

Of course, the same considerations hold true if the driver $f$ is allowed to depend on a control parameter and the control process is predictable. In fact, more general drivers depending on $\omega$ can be considered and the arguments remain identical. Finally, the assumption $\left(\mathrm{A}_{O}\right)$ hints to the fact that we are going to work with bounded $y$. We have also introduced a state $\Delta$ to describe a cemetery state for the second component. This state can be taken to be in $\mathbb{R}^{m}$, far from the domains in $\left(\mathrm{A}_{O}\right)$ and (by eventually modifying its values), we assume $f$ to be zero at $y=\Delta$. 


\subsubsection{A Scheme Based On Reflected Solutions for Ordinary Differential Equations}

We consider a càdlàg process $Y$ continuous except, maybe, at switching times $T_{n}$. Then, as explained before, this can be identified with a family $\left(y^{n}\right)$. At jumping times $T_{n+1}$, the process $Y$ is something like

$$
Y_{T_{n+1}}=y^{n+1}\left(e_{n} \oplus\left(T_{n+1}, \Gamma_{T_{n+1}}\right), T_{n+1}\right) .
$$

We construct, for every $n \geq 0$,

$$
\widehat{y}^{n+1}(e, t, \gamma):=y^{n+1}(e \oplus(t, \gamma), t) 1_{|e|<t}
$$

and $Y_{T_{n+1}}$ can be obtained by simple integration of the previous quantity with respect to the conditional law of $\left(T_{n+1}, \Gamma_{T_{n+1}}\right)$ knowing $\mathcal{F}_{T_{n}}$.

We introduce the following scheme. We let $\xi$ be a final condition. We "correct" $\xi=\left(\xi^{n}\right)$ given by (5) as to be in the admissible domains as follows:

$$
\xi_{a d m}^{n}(e):=\xi^{n}(e) 1_{\xi^{n}(e) \in \mathcal{O}_{\gamma_{|e|}}}+\Delta 1_{\xi^{n}(e) \in \mathcal{O}_{\gamma_{|e|}^{c}}^{c}} .
$$

It is obvious that, should the data not be in the target domain, there is no point in solving the reflected BSDE. In this case, we simply set the solution to be a constant point $\Delta$ designed to be a flag signaling that infection cannot precede the current time.

We consider the family of (ordinary) differential inclusions

$$
\left\{\begin{aligned}
& y^{M}\left(e_{M}(\omega), t\right)=\xi_{a d m}^{M}\left(e_{M}(\omega)\right), \\
& \text { For } n \leq M-1, \xi_{a d m}^{n,+}\left(e_{n}(\omega)\right):=\left\{\begin{aligned}
\xi_{a d m}^{n}\left(e_{n}(\omega)\right), & \text { if } y^{n+1}\left(e_{n+1}(\omega), 0\right) \in \mathcal{O}_{\gamma_{\left|e_{n}(\omega)\right|}}, \\
\Delta, & \text { otherwise }
\end{aligned}\right. \\
&-d y^{n}\left(e_{n}(\omega), t\right)+H\left(t, y^{n}\left(e_{n}(\omega), t\right)\right) \partial^{-} \varphi_{\mathcal{O}_{\gamma_{\mid e_{n}}(\omega) \mid}}\left(y^{n}\left(e_{n}(\omega), t\right)\right) d t \ni \\
& \quad+\sum_{\gamma \in E} f\left(\widehat{y}^{n+1}\right)^{n}\left(e_{n}(\omega), s, \gamma, y^{n}\left(e_{n}(\omega), s\right),-y^{n}\left(e_{n}(\omega), s\right)\right) \widehat{q}_{e_{n}(\omega)}^{n}(d s,\{\gamma\}), \\
& y^{n}\left(e_{n}(\omega), T\right)=\xi_{a d m}^{n,+}\left(e_{n}(\omega)\right) .
\end{aligned}\right.
$$

Let us assume, for the time being, that this system admits an unique solution. Since the assumptions $\left(\mathrm{A}_{O}, \mathrm{~A}_{F}\right)$ hold true, the existence and uniqueness of the solution for (13) reduce to a generic problem for each constituent equation from (13). The exact proof of this claim concerning the component equations will be given shortly after. The following result links the solvability of the initial reflected problem and the (finite) system of (backward) ordinary differential equations (13).

Proposition 7 Let us assume that $\left(A_{O}, A_{H}, A_{F}\right.$ and $\left.A_{M}\right)$ hold true. Then, the càdlàg process $Y=\left(y^{n}\right)$ continuous, except at switching times, is a solution for (1) if and only if it satisfies the system (13).

The proof is inspired by the non-reflected version in [16]. The elements of proof are provided in Section 5. The basic idea is to employ the structure presented in the previous subsection. Indeed, since $Z$ only acts at jumping times, there is a simple relation linking $z^{n}$ to $y^{n}$ and $\widehat{y}^{n+1}$. The conclusion follows by plugging this $z$ into the equation.

\subsubsection{The Iterating Differential Inclusion}

As we have seen in Proposition 7, the BSVI can be reduced to a family of ordinary differential equations in which the starting data (given at final time) is given iteratively. Therefore, in this subsection we turn our attention to the solvability of such reflected differential inclusions. To 
this purpose, let us freeze the regular domain $\mathcal{O}$ of a $(\rho, \beta)$-semiconvex function $\varphi_{\mathcal{O}}$ satisfying the assumption $\left(\mathrm{A}_{O}\right)$. The inclusion has the form:

$$
\left\{\begin{array}{l}
-d y(t)+H(t, y(t)) \partial^{-} \varphi_{\mathcal{O}}(y(t)) d t \ni \int_{E} \bar{f}\left(t, \gamma^{\prime}, y(t)\right) \nu\left(d t, d \gamma^{\prime}\right), \\
y(T)=\eta
\end{array}\right.
$$

where $\nu$ stands for the compensator $\widehat{p}$. Throughout the remaining of the section and unless stated otherwise, the function $\bar{f}: \mathbb{R}_{+} \times E \times \mathbb{R}^{m} \longrightarrow \mathbb{R}^{m}$ is assumed to be globally continuous, bounded and Lipschitz continuous in space, uniformly with respect to the time and $\gamma \in E$. Moreover, due to the particular form of our $\widehat{p}$, the measure $\nu$ is assumed to be positive, and $\nu(d t, E)$ to have a bounded Radon-Nikodym derivative with respect to the Lebesgue measure on $[0, T]$.

Let us now give a precise definition of the notion of solution we are going to employ in connection to the previous ordinary differential inclusion.

Definition 8 A solution of (14) consists of a couple $(y, k)$ satisfying simultaneously the following:

(i) 1. The function $y \in C\left([0, T] ; \mathbb{R}^{m}\right)$ is continuous and for Leb -almost all $t, y(t) \in \mathcal{O}$.

2. The application $[0, T] \ni t \mapsto \varphi_{\mathcal{O}}(y(t))$ is integrable w.r.t. Lebesgue measure.

(ii) 1. The function $k \in \mathbb{L}^{2}\left([0, T] ; \mathbb{R}^{m}\right)$ is square integrable w.r.t. Lebesgue measure.

2. For Leb-almost all $t \in[0, T]$, one has $k(t) \in \partial^{-} \varphi_{\mathcal{O}}(y(t))$.

(iii) The equality $y(t)+\int_{t}^{T} H(s, y(s)) k(s) d s=\eta+\int_{t}^{T} \int_{E} \bar{f}\left(s, \gamma^{\prime}, y(s)\right) \nu\left(d s, d \gamma^{\prime}\right)$, holds true, Leb-almost everywhere.

Remark 9 Condition (ii) is equivalent to asking that, for every, $0 \leq s \leq t \leq T$ and every $\mathbb{R}^{m}$-valued, continuous function $x \in C\left([0, T] ; \mathbb{R}^{m}\right)$, one has

$$
\begin{aligned}
\int_{s}^{t}\langle x(r)-y(r), k(r)\rangle d r+\int_{s}^{t} \varphi_{\mathcal{O}}(y(r)) d r \\
\quad \leq \int_{s}^{t} \varphi_{\mathcal{O}}(x(r)) d r+\int_{s}^{t}|x(r)-y(r)|^{2}(\rho+\beta|k(r)|) d r .
\end{aligned}
$$

For further details, the reader is referred to [41].

The following result gives the existence and uniqueness of solutions to the deterministic equation (14).

Theorem 10 We assume $\left(A_{O}\right)$ and $\left(A_{H}\right)$ to hold true (where $\mathcal{O}_{\gamma}$ is replaced with $\mathcal{O}$ ). Then, for every $\eta \in \mathcal{O}$, there exists an unique couple of deterministic functions $(y, k) \in C\left([0, T] ; \mathbb{R}^{m}\right) \times$ $\mathbb{L}^{2}\left([0, T] ; \mathbb{R}^{m}\right)$ which satisfies (14), in the sense of Definition 8.

The proof relies on the penalization of the multivalued operator $\partial^{-} \varphi_{\mathcal{O}}$. For our readers' sake, we give the main arguments in Section 5. The basic idea is to start with providing convenient estimates on the approximating solutions. The estimates are much like those in [41, Theorems 7 and 8 ] and they provide local solutions. For our bounded framework, the solution and estimates are global. To conclude, one passes to the limit on these penalized equations. Both the estimates and the method employed to prove the result are of particular relevance for the next section.

\section{Targeted Design}

In the previous paragraphs, the jump mechanism has been constructed starting from given $\lambda$ and $Q$ and with a given driver $f$. In our phage model, this hybrid mechanism is essentially governed by 
the reaction speeds $k$. Nevertheless, these speeds can be altered in various ways. To give a simple example, the reaction constants in [17] in simulations vary with the choice of the product half time denoted $T p\left(1 \mathrm{~h}, 2 \mathrm{~h}, 4 \mathrm{~h}\right.$, etc.) and the stability of the regime (e.g. $\frac{4 \ln 2}{T p}$ for slow-unstable, $\frac{40 \ln 2}{T p}$ for fast-unstable, etc.).

Therefore, we consider our differential mechanism to be governed by an exogenous control parameter that can be associated to temperature and/or catalysts conditions. Then, the construction of the differential component is given by a driver depending on a predictable control process $u$. From now on, we let $U$ be a compact metric space and assume that the driver function $f: \mathbb{R}_{+} \times E \times E \times \mathbb{R}^{m} \times \mathbb{R}^{m} \times U \longrightarrow \mathbb{R}^{m}$ is globally continuous, bounded and there exists some constant $c>0$ such that

$$
\left|f\left(t, \gamma, \gamma^{\prime}, y, z, u\right)-f\left(t, \gamma, \gamma^{\prime}, y^{\prime}, z^{\prime}, u\right)\right| \leq c\left(\left|y-y^{\prime}\right|+\left|z-z^{\prime}\right|\right)
$$

for all $\left(t, \gamma, \gamma^{\prime}, y, y^{\prime}, z, z^{\prime}, u\right) \in \mathbb{R}_{+} \times E \times E \times \mathbb{R}^{4 m} \times U$. To show the relevance of our approach, let us turn our attention to a simple example.

\subsection{A Simplified Workout Example}

Starting from the initial system, we consider the linearized version of the deterministic dynamics by leaving aside the quadratic term and introduce a control parameter $u$ to govern transcription :

$$
\left\{\begin{array}{l}
\frac{d X_{1}}{d t}=f_{1}\left(\Gamma_{t}, X, u\right):=-k_{d} X_{1}+2 k_{-1} X_{2}+R u 1_{\Gamma_{t}=e_{2}} \\
\frac{d X_{2}}{d t}=f_{2}\left(\Gamma_{t}, X\right):=-k_{-1} X_{2}
\end{array}\right.
$$

Boundedness of the Domain(s). We pick $k_{d}=3, k_{-1}=k_{ \pm 3}=k_{ \pm 4}=1, R=1, u \in[0,1]$. It is easy to see that, for all $\left(x_{1}, x_{2}\right) \in[0,1]^{2}$,

$$
f_{1}\left(\gamma,\left(\begin{array}{c}
1 \\
x_{2}
\end{array}\right), u\right) \leq 0, f_{1}\left(\gamma,\left(\begin{array}{c}
0 \\
x_{2}
\end{array}\right), u\right) \geq 0, f_{2}\left(\gamma,\left(\begin{array}{c}
x_{1} \\
0
\end{array}\right), u\right)=0, f_{2}\left(\gamma,\left(\begin{array}{c}
x_{1} \\
1
\end{array}\right), u\right) \leq 0 .
$$

It is, therefore, obvious that the set $[0,1]^{2}$ is forward-invariant. An alternative to these considerations is to apply invariance results (given w.r.t. normal cones in [28], for instance).

To simplify the arguments, we assume that the lysogenic domains are mode-independent and given by $\mathcal{O}:=\left\{\left(x_{1}, x_{2}\right) \in[0,1]: x_{1}^{2}+x_{2}^{2} \geq 1 / 25\right\}$. We also consider normal reflection.

Minimal and Maximal Transcription. When $u=0$, it is clear that the (unreflected) couple repressor/dimer is given by $X_{t}=\left(\begin{array}{cc}e^{3(T-t)} & e^{(T-t)}-e^{3(T-t)} \\ 0 & e^{(T-t)}\end{array}\right) X_{T}$. A simple glance at the eigenvalues leads to the conclusion that the dynamics are expansive in the sense that the distance to the origin increases and exceeds $\left|X_{T}\right|$. Hence, any solution which starts (at time $T$ ) in $\mathcal{O}$ can never get to the lysis region $\left\{\left(x_{1}, x_{2}\right) \in[0,1]: x_{1}^{2}+x_{2}^{2}<1 / 25\right\}$. However, according to our model, the solution can exist only for as long as $\left|X_{t}\right| \leq 1$. The reflection will keep the solution in $\mathcal{O}$ but the time of infection in this case is obtained by observing the "occupation" of the frontier $\left|x_{1}\right| \vee\left|x_{2}\right|=1$. Moreover, except for deterministic final data, the BSDE has a non-zero $Z$. This is rather obvious, since, for this choice of $u$, the system does not change the vector field at switches.

For $u=1$, let us consider the case of (at most) two jumps starting from the DNA configuration $\gamma_{0}=1$. Our DNA model gives a transition matrix

$$
Q=\left(\begin{array}{cccc}
0 & \frac{1}{2} & \frac{1}{2} & 0 \\
\frac{1}{2} & 0 & 0 & \frac{1}{2} \\
1 & 0 & 0 & 0 \\
0 & 1 & 0 & 0
\end{array}\right)
$$


It is, therefore, obvious that the law of $\Gamma_{1}$ under $\mathbb{P}^{1}$ is given by $\frac{1}{2} \delta_{2}+\frac{1}{2} \delta_{3}$. Solving the backward equation on $\left(T_{2}, T\right]$ from a deterministic final data $\eta \in \mathcal{O}$ gives (thanks to Proposition 7 ), $\circ \xi^{0}=\xi^{1}=\xi^{2}=\eta, y^{2}\left(e_{2}, \cdot\right)=\eta, \xi_{a d m}^{1,+}=\eta$.

○ On $\Gamma_{1}=2, y^{1}\left(e_{1}, t\right)$ is the solution of the reflected equation and, prior to $t_{0}$, which is the hitting time of $\overline{\mathbb{B}}(0,1 / 5), y^{1}\left(e_{1}, t\right)=\left(\begin{array}{c}\left(\eta_{1}-\eta_{2}-\frac{1}{3}\right) e^{3(T-t)}+\eta_{2} e^{T-t}+\frac{1}{3} \\ \eta_{2} e^{T-t}\end{array}\right) 1_{t \geq t_{0}}$.

○ On $\Gamma_{1}=3, y^{1}\left(e_{1}, t\right)=\left(\begin{array}{c}\left(\eta_{1}-\eta_{2}\right) e^{3(T-t)}+\eta_{2} e^{T-t} \\ \eta_{2} e^{T-t}\end{array}\right)$.

- Finally, $y^{0}(1, \cdot)$ has the same expression as $y^{1}$ on $\Gamma_{1}=3$.

For obvious reasons (the reflected solution being nonlinear), we do not compute this solution explicitly but give the comparison between the reflected form and the one in which reflection does not occur. The numerical approximation of $y^{1}$ on $\Gamma_{1}=2$ is illustrated in Fig. 3. Note that transcription is at high level. Thinking backwards, the only possibility for the repressor not to reach the interior of lytic domain is to be compensated by the dimer. For our choice of parameters, the solution is kept (locally in time) on the boundary (until it can be pushed in the interior of the lysogenic domain).

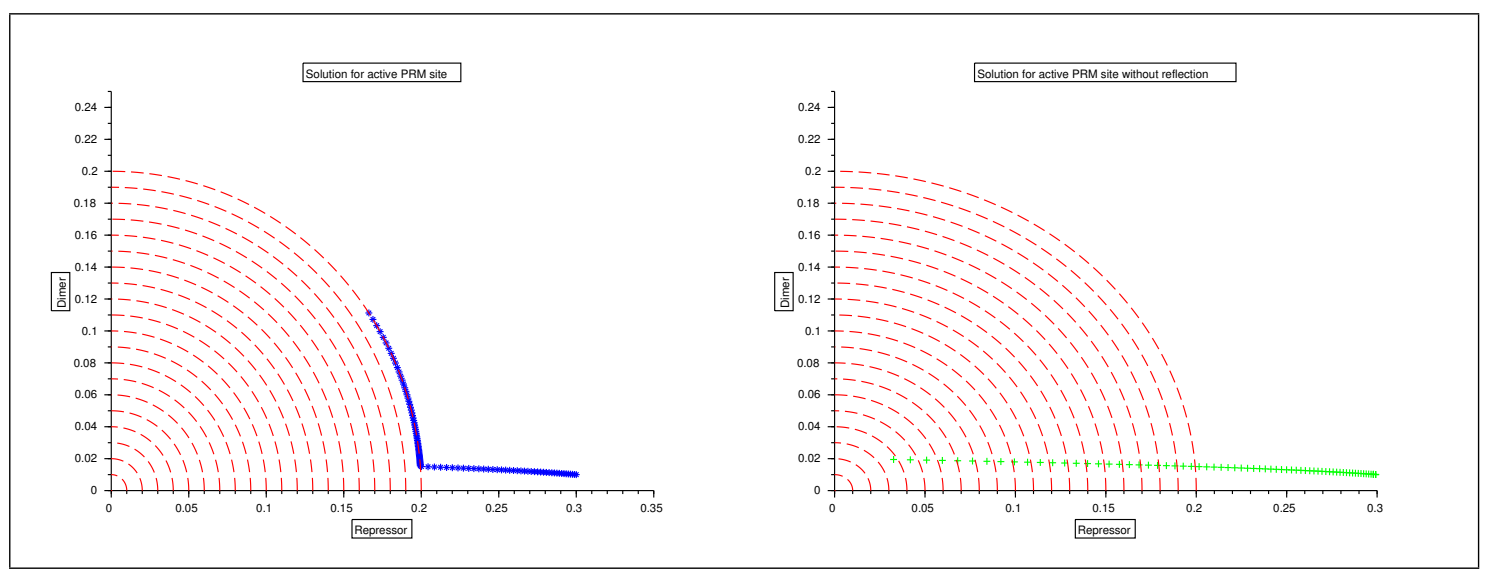

Fig 3. Reflected and non-reflected solution for PRM (2)

Comments. In both cases, there are some drawbacks. In the first case $(u=0)$, the $Z$ component is non-zero and, hence, we do not actually get a continuously switching protein concentration. In the second one, the reflected solution has a "high concentration" on the boundary of the lytic domain. This means that alert flags are triggered at host level which should, normally lead to excision (instead of the equation we have solved backwards). These case studies show that an equilibrium should be envisaged concerning transcription. In other words, we should pick "the best" $u$ by convexifying the control space (at least by allowing $u \in[0,1]$ ) and looking at the time and space occupied by $Z$ and/or $Y$ on the frontier of the domain.

\subsection{Occupation measures}

Using the previous intuition, we are going to introduce the linear programming formulations in connection to these problems. For more details on the subject and the link between the linear formulations and the classical control problems in forward dynamics, the reader is referred to [24] (deterministic setting), [6], [9], [36], [37], [42], [45] [12] (various stochastic settings), [23], [29] or [18] (for the more general PDMP processes). To our best knowledge, although extensively employed in forward problems, this is the first time one employs occupation measures in connection to backward problems. 
To fully understand the considerations of this section, the reader is invited to take a look at the proof of Theorem 10. The basic idea is the following. To solve the BSVI, one replaces $\varphi$ with inf-convolutions of type $\varphi^{\varepsilon}(x):=\inf _{y \in \mathbb{R}^{m}}\left\{\frac{1}{2 \varepsilon}|x-y|^{2}+\varphi(y)\right\}$, for $x \in \mathbb{R}^{m}$ and $\varepsilon>0$. To every such penalized solution corresponding to a predictable control, one can associate a measure taking into account all the components: time, accessible mode, occupation of the space (given by $Y$ ) and corrective term $(Z)$ as well as the control. A further variable takes into account the gradient. These measures are shown (in the proof of Proposition 11) to satisfy convenient compactness criteria and exhibit a support condition related to the subgradient. Itô's formula provides a linear-type restriction of these measures. Finally, to facilitate passage to the limit in penalizations, we give a (support) condition related to the distance to the lysogeny domains.

The reader is invited to note that the solution $Y^{T, \xi, u}$ belongs to $\mathcal{O}:=\left(\cup_{\gamma \in E} \mathcal{O}_{\gamma}\right) \cup\{\Delta\}$. Therefore, under the boundedness assumption on the domains, $Y_{t}^{T, \xi, u} \in \overline{\mathbb{B}}(0, C) \cup\{\Delta\}$, for some generic constant $C$. This constant can be chosen independent of the penalization and is given by our data (cf. Remark 15). Similar assertions hold true for the $Z$ component. To see this, it is necessary to look at (15) in the next section. Motivated by the approach in the forward setting (cf. [29]) as well as the singular perturbations setting in [30], we introduce the following sets

$$
\begin{aligned}
& E^{\Delta, t, T}:=[t, T] \times E^{2} \times\left(\left(\overline{\mathbb{B}}(0, C) \times \mathbb{R}^{m}\right) \cup(\Delta \times\{0\})\right) \times \overline{\mathbb{B}}(0,2 C+|\Delta|) \\
& \Theta_{\varepsilon}(t, T, \xi):= \\
& \left\{\begin{array}{l}
\mu \in \mathcal{P}(\overline{\mathbb{B}}(0, C) \cup\{\Delta\}) \times \mathcal{P}\left(E^{\Delta, t, T} \times U\right), \\
\forall \phi \in C_{b}^{1,2}\left([t, T] \times \mathbb{R}^{m}\right), \\
\mathbb{E}^{\gamma_{0}}[\phi(T, \xi)]=\int_{\mathbb{R}^{m}} \phi(t, y) \mu^{1}(d y) \\
\quad+\int_{E^{\Delta, t, T}}\left\langle\nabla_{y} \phi\left(s, y_{1}\right), H\left(s, y_{1}\right) y_{2}\right\rangle \mu^{2}\left(d s d \gamma^{\prime} d \gamma d y_{1} d y_{2} d z, U\right) \\
\quad-\int_{E^{\Delta, t, T} \times U}\left\langle\nabla_{y} \phi\left(s, y_{1}\right), f\left(s, \gamma^{\prime}, \gamma, y_{1}, z, u\right) \lambda(\gamma) Q\left(\gamma,\left\{\gamma^{\prime}\right\}\right)\right\rangle \mu^{2}\left(d s d \gamma^{\prime} d \gamma d y_{1} d y_{2} d z d u\right) \\
\quad+\int_{E^{\Delta, t, T}}\left(\partial_{t} \phi\left(s, y_{1}\right)+\phi\left(s, y_{1}+z\right)-\phi\left(y_{1}\right)\right) \lambda(\gamma) Q\left(\gamma,\left\{\gamma^{\prime}\right\}\right) \mu^{2}\left(d s d \gamma^{\prime} d \gamma d y_{1} d y_{2} d z, U\right), \\
S u p p\left(\mu^{2}\right) \subset\left\{\begin{array}{l}
\left(s, \gamma^{\prime}, \gamma, y_{1}, y_{2}, z, u\right): \forall a \in \mathbb{R}^{m}, \\
\left.\left\langle a-y_{1}, y_{2}\right\rangle+\varphi_{\mathcal{O}_{\gamma}}^{\varepsilon}\left(y_{1}\right) \leq \varphi_{\mathcal{O}_{\gamma}}^{\varepsilon}(a)+\left(\rho+\beta\left|y_{2}\right|\right)\left|a-y_{1}\right|^{2}\right\} \\
\int_{E^{\Delta, t, T}}\left(d_{\mathcal{O}_{\gamma}}^{2} \wedge 1\right)\left(y_{1}\right) \mu^{2}\left(d s d \gamma^{\prime} d \gamma d y_{1} d y_{2} d z, U\right) \leq C \varepsilon \\
\int_{E^{\Delta, t, T}}\left|y_{2}\right|^{2} \mu^{2}\left(d s d \gamma^{\prime} d \gamma d y_{1} d y_{2} d z, U\right) \leq C .
\end{array}\right.
\end{array}\right.
\end{aligned}
$$

The link between these sets and the actual solution of our initial problems will appear explicitly in the proofs. For now, all one needs to know is the following.

Proposition 11 Let us fix $\varepsilon>0$, the time horizon $T>0,0 \leq t \leq T$ and the final data $\xi$. Then, the family $\Theta_{\varepsilon}(t, T, \xi)$ is non-empty, convex and compact (with respect to the usual topology on the space of probability measures).

As explained before, we embed the solutions of our (approximating) BSDE into a measure. The linear restriction is a mere reformulation of Itô's formula. The support condition is linked to gradients. The distance to lysogeny domains follows from the estimates on approximating solutions as do the second order moments (guaranteeing compactness). We postpone the proof to Section 5 .

Second, following the approximating construction of solution to the initial problem (1) (see proof of Theorem 10), one considers the lower limit of sets

$$
\Theta_{0}(t, T, \xi)=\liminf _{\varepsilon \rightarrow 0} \Theta_{\varepsilon}(t, T, \xi) .
$$

Admit, for the time being (the actual proof is given afterwards) that the solutions to the initial BSVI (with control) can be seen as elements of the limit set $\Theta_{0}(t, T, \xi)$. Then one is entitled to ask oneself if these solutions also enjoy similar properties (regularity, support and linear-type restriction). This is, indeed, the case as summarized by the following result. 
Theorem 12 (i) (convexity and compactness) The set $\Theta_{0}(t, T, \xi)$ is a non-empty, convex and compact subset of $\mathcal{P}(\overline{\mathbb{B}}(0, C) \cup\{\Delta\}) \times \mathcal{P}\left(E^{\Delta, t, T} \times U\right)$ and, for every $\mu=\left(\mu^{1}, \mu^{2}\right) \in \Theta_{0}(t, T, \xi)$,

$$
\int_{E^{\Delta, t, T} \times U}\left|y_{2}\right|^{2} \mu^{2}\left(d s d \gamma^{\prime} d \gamma d y_{1} d y_{2} d z d u\right) \leq C .
$$

(ii) (support and subdifferential) Every measure $\mu=\left(\mu^{1}, \mu^{2}\right) \in \Theta_{0}(t, T, \xi)$ satisfies the support condition

$$
\operatorname{Supp}\left(\mu^{2}\right) \subset\left\{\left(s, \gamma^{\prime}, \gamma, y_{1}, y_{2}, z, u\right): y_{1} \in \mathcal{O}_{\gamma}, y_{2} \in \partial^{-} \varphi_{\mathcal{O}_{\gamma}}\left(y_{1}\right)\right\} .
$$

(iii) (linear constraint) Every limit measure $\left(\mu^{1}, \mu^{2}\right) \in \Theta_{0}(t, T, \xi)$ satisfies

$$
\begin{aligned}
& \mathbb{E}^{\gamma_{0}}[\phi(T, \xi)] \\
& \in \int_{\mathbb{R}^{m}} \phi(t, y) \mu^{1}(d y) \\
& \quad+\liminf _{\varepsilon \rightarrow 0}\left\{\int_{E^{\Delta, t, T} \times U}\left\langle\nabla_{y} \phi\left(s, y_{1}\right), H\left(s, y_{1}\right) y_{2}\right\rangle \eta^{2}\left(d s d \gamma^{\prime} d \gamma d y_{1} d y_{2} d z d u\right): \eta \in \Theta_{\varepsilon}(t, T, \xi)\right\} \\
& \quad-\int_{E^{\Delta, t, T} \times U}\left\langle\int_{E} f\left(s, \gamma^{\prime}, \gamma, y_{1}, z, u\right) \lambda(\gamma) Q\left(\gamma, d \gamma^{\prime}\right)\right\rangle \mu^{2}\left(d s d \gamma^{\prime} d \gamma d y_{1} d y_{2} d z d u\right) \\
& \quad+\int_{E^{\Delta, t, T} \times U}\left(\partial_{t} \phi\left(s, y_{1}\right)+\phi\left(s, y_{1}+z\right)-\phi\left(y_{1}\right)\right) \lambda(\gamma) Q\left(\gamma,\left\{\gamma^{\prime}\right\}\right) \mu^{2}\left(d s d \gamma^{\prime} d \gamma d y_{1} d y_{2} d z d u\right) .
\end{aligned}
$$

Remark 13 (i) This kind of relaxation has been recently employed in order to characterize Pontryagin-type optimality criteria in forward Brownian settings. To this purpose, the interested reader is referred to [30].

(ii) In the convex setting, whenever the functions $\varphi_{\mathcal{O}}$ have at most quadratic growth, using the gradient estimates in [27, Proposition 4.3], one gets $\sup _{y_{2} \in \partial \varphi_{\mathcal{O}}\left(y_{1}\right)}\left|y_{2}\right| \leq c\left(1+\left|y_{1}\right|\right)$, for some constant $c>0$. In this framework, one can replace

$$
\liminf _{\varepsilon \rightarrow 0}\left\{\int_{E^{\Delta, t, T} \times U}\left\langle\nabla_{y} \phi\left(s, y_{1}\right), H\left(s, y_{1}\right) y_{2}\right\rangle \eta^{2}\left(d s d \gamma^{\prime} d \gamma d y_{1} d y_{2} d z d u\right): \eta \in \Theta_{\varepsilon}(t, T, \xi)\right\}
$$

with the term

$$
\int_{E^{\Delta, t, T} \times U}\left\langle\nabla_{y} \phi\left(s, y_{1}\right), H\left(s, y_{1}\right) y_{2}\right\rangle \mu^{2}\left(d s d \gamma^{\prime} d \gamma d y_{1} d y_{2} d z d u\right) .
$$

Yet another way of writing the linear constraint condition would be to study the asymptotic behavior of the $\mathbb{R}^{m}$-valued vector measures with uniformly-bounded variation

$$
y_{2} \mu^{\varepsilon, 2}\left(d s d \gamma^{\prime} d \gamma d y_{1} d y_{2} d z d u\right) \text {, where } \mu^{\varepsilon}=\left(\mu^{\varepsilon, 1}, \mu^{\varepsilon, 2}\right) \in \Theta_{\varepsilon}(t, T, \xi) .
$$

The proof of the theorem is postponed to Section 5 .

Conclusions To conclude the section, in order to design the best fitting model for our pathogen/ host system, one could proceed as follows. First, identify the desired target behavior (i.e. $\xi$ ). Next, among the (relaxed) measures in $\Theta_{0}(t, T, \xi)$, select those who best fit your purpose. To come back to the comments on our linearized model, if one wishes to avoid jumps on the protein concentration, one has to minimize $\int_{E^{\Delta, t, T} \times U}|z| \mu^{2}\left(d s d \gamma d y_{1} d y_{2} d z d u\right)$ (recall that continuous switch is given by $z=0$ support). To get the largest activity (lysogeny) time, one maximizes $\int_{E^{\Delta, t, T} \times U} s 1_{y_{1} \neq \Delta} \mu^{2}\left(d s d \gamma d y_{1} d y_{2} d z d u\right)$. To maximize coherence of the model, one should occupy the boundary of the lysogenic domain as little as possible, which leads to minimizing the quantity $\int_{E^{\Delta, t, T} \times U} 1_{y_{1} \in B d\left(\mathcal{O}_{\gamma}\right)} \mu^{2}\left(d s d \gamma d y_{1} d y_{2} d z d u\right)$. These problems exceed the purpose of the present article. However, since we have nice regularity of the constraints domain $\Theta_{\varepsilon}(t, T, \xi)$, one simply 
passes to the Fenchel dual (see [28] or [30] for forward PDMP or singular perturbations settings). Optimality conditions can then be obtained as in [30].

However, while in the forward case these sets of constraints are shown to be the closed convex hulls of occupation measures, this is less known under the present framework. We are still searching a method adapted to this case (Krylov-like shaking the coefficients followed by mollification arguments as in the forward case is envisaged).

Further generalizations may benefit from the piecewise diffusive switched framework in [35] (replacing the approach in [16]). This would allow treating mesoscopic models in which a secondorder approximation (based on the central limit theorem instead of the law of large numbers) appears (similar to [3]). Nevertheless, technical difficulties appear on the predictable component of the inter-jumps Brownian driven BSVI and global estimates (as in proof of Theorem 10) are far from obvious.

Finally, we wish to emphasize that the special assumptions on the mode component $(\Gamma)$ allows one to use the techniques of [16] and reduce the BSVI to a system of ordinary differential inclusions. This is a key point for the proofs. In all generality, the jump parameter $\lambda$ is computed as a propensity function and may depend on all the components (see, for example, [20]). In this case, the Marked point mechanism is quite different changing with the control input and one should use a relaxed framework.

\section{Proofs of the Results in Sections 3 and 4}

This section gathers all the proofs of the results in Sections 3 and 4 .

\subsection{Proof of Proposition 7}

We begin with providing the elements of proof for the equivalence between the BSVI (1) and the system of ordinary differential inclusions (13). The proof strongly relies on the structure properties mentioned in Subsection 3.2.1. The idea is to associate a specific form to the jump component $Z^{T, \xi}$ and plug it into the driver written as in Subsection 3.2.1.

Proof of Proposition 7. We begin our proof with noting that if $Y^{T, \xi}=\left(y^{n}\right)$ is a solution to the initial system (1), then the jumps only occur at times $T_{n} \leq T$ and

$$
Y_{T_{n}}^{T, \xi}-Y_{T_{n}-}^{T, \xi}=Z_{T_{n}}^{T, \xi}\left(\Gamma_{T_{n}}\right) .
$$

On the other hand, for every $n \leq M-1$, recalling (11), it follows that

$$
Z_{T_{n+1}}^{T, \xi}\left(\Gamma_{T_{n+1}}\right)=y^{n+1}\left(e_{n} \oplus\left(T_{n+1}, \Gamma_{T_{n+1}}\right), T_{n+1}\right)-y^{n}\left(e_{n}, T_{n+1}\right) .
$$

This equality, as well as those following are understood as everywhere except a $\mathbb{P}^{\gamma_{0}}$-null set. Since we will be dealing with a finite family (for $n \leq M$ ), taking the union of such sets gives us yet another $\mathbb{P}^{\gamma_{0}}$-null set. We recall that the process $Z^{T, \xi}$ has to be progressively measurable such that a version of the process $Z^{T, \xi}$ is defined by setting

$$
z^{n}(e, t, \gamma):=y^{n+1}(e \oplus(t, \gamma), t) 1_{t>|e|}-y^{n}(e) .
$$

It is then obvious (see also [16]) that, on the stochastic interval $s \in\left(T_{n}, T_{n+1}\right]$, one has

$$
f\left(s, \gamma, \Gamma_{s-}, Y_{s-}^{T, \xi}, Z_{s}^{T, \xi}\right)=f\left(\widehat{y}^{n+1}\right)^{n}\left(s, \gamma, y^{n}\left(e_{n}, s\right),-y^{n}\left(e_{n}, s\right)\right),
$$

where $\widehat{y}^{n+1}$ is given by (12).

We prove that any solution of the BSVI (1) satisfies the system of ordinary differential inclusions (13). The converse is quite similar and makes use of the same elements of proof. If $n=M$, one has 
$T_{n+1}=\infty, \mathbb{P}^{\gamma_{0}}$-almost everywhere on $\Omega$ and the compensator satisfies the $(\infty, \bar{\gamma})$-support condition in (8). This easily implies the terminal condition on $y^{M}$.

On $T_{n} \leq t<T_{n+1}$, one has

$$
Y_{t}^{T, \xi}+\int_{t}^{T_{n+1} \wedge T} K_{s}^{T, \xi} d s=Y_{T_{n+1}-}^{T, \xi} 1_{T_{n+1} \leq T}+\xi 1_{T_{n+1}>T}+\int_{t}^{T_{n+1} \wedge T} f\left(s, \gamma, \Gamma_{s-}, Y_{s-}^{T, \xi}, Z_{s}^{T, \xi}\right) \widehat{p}(d s, d \gamma)
$$

if $Y_{T_{n+1}-}^{T, \xi} \neq \Delta$. If $Y_{T_{n+1}-}^{T, \xi}=\Delta$, then, by definition, $\left(Y_{t}^{T, \xi}, K_{t}^{T, \xi}\right)=(\Delta, 0)$ on $t<T_{n+1}$. Moreover, $f(\cdot, \cdot, \cdot, \Delta, \cdot)$ is null such that the previous equality is trivially satisfied. Then, except on a $\mathbb{P}^{\gamma_{0}}$-null set, recalling that we have introduced (15), (12) and using the notations in Section 3.2.1, one gets

$$
\begin{aligned}
y^{n}\left(e_{n}(\omega), t\right)+\int_{t}^{T_{n+1}(\omega) \wedge T} H\left(s, y^{n}\left(e_{n}(\omega), s\right)\right) k^{n}\left(e_{n}(\omega), s\right) d s \\
=y^{n}\left(e_{n}(\omega), T_{n+1}(\omega)\right) 1_{T_{n+1}(\omega) \leq T}+\xi^{n}\left(e_{n}(\omega)\right) 1_{T_{n+1}(\omega)>T} \\
\quad+\int_{t}^{T_{n+1}(\omega) \wedge T} \sum_{\gamma \in E} f\left(\widehat{y}^{n+1}\right)^{n}\left(s, \gamma, y^{n}\left(e_{n}(\omega), s\right),-y^{n}\left(e_{n}(\omega), s\right)\right) \widehat{q}_{e_{n}(\omega)}^{n}(d s,\{\gamma\}) .
\end{aligned}
$$

(The reader will want to note that $Y_{T_{n+1}-}^{T, \xi} \neq \Delta$ implies that $Y_{t}^{T, \xi} \neq \Delta$ on $T_{n+1} \leq t$.) We can actually write this equality for every $t$. Indeed, due to (6), (5) one freezes $y^{n}$ for $t \leq T_{n}(\omega)$ and everything is set to 0 if $T<T_{n}(\omega)$ i.e.

$$
\left\{\begin{array}{l}
y^{n}\left(e_{n}(\omega), t\right)=y^{n}\left(e_{n}(\omega), T_{n}(\omega)\right), \text { if } t \leq T_{n}(\omega) \text { and } \\
\xi^{n}\left(e_{n}(\omega), t\right)=y^{n}\left(e_{n}(\omega), t\right)=0, \text { whenever } T<T_{n}(\omega)
\end{array}\right.
$$

and recall the support conditions on the compensator (8). This gives our claim.

\section{2 (Elements of) Proof of Theorem 10}

To prove Theorem 10, one uses a penalizing approach similar to the classical Moreau-Yosida-Brézis one for the convex context. For more details, the reader is referred to the comprehensive studies in [11] or [4]. Recently, the authors of [41] have developed a penalization approach to multivalued differential equations with generalized reflection in a nonconvex setting.

The equations that make the object of our framework (and those appearing in [41]) cannot be tackled by the semigroup operators theory because of the particular structure of the multivalued term. Indeed, mixing a reflection matrix and a monotone operator (such as the subdifferential) leads to losing both monotonicity and Lipschitz properties of its constituent parts.

Whenever $\varphi: \mathbb{R}^{m} \rightarrow(-\infty,+\infty]$ is a lower semicontinuous function such that, for some $a, b, c \geq 0$ one has

$$
\varphi(y)+a|y|^{2}+b|y|+c \geq 0,
$$

for all $y \in \mathbb{R}^{m}$, we introduce, for $\varepsilon>0$, the usual inf-convolution

$$
\varphi^{\varepsilon}(x):=\inf _{y \in \mathbb{R}^{m}}\left\{\frac{1}{2 \varepsilon}|x-y|^{2}+\varphi(y)\right\},
$$

for all $x \in \mathbb{R}^{m}$. The following proposition summarizes the main properties of inf-convolutions in a semiconvex setting. These results are borrowed from [41] and will turn out to be very useful in the study of (14). To summarize, one gets a neighborhood of the domain of $\varphi$ on which the infimum giving the inf-convolution is attained. The minimizing argument is unique and belongs to $\operatorname{Dom}(\varphi)$. Convenient local estimates on the gradient of the penalization are given as well as links with the Fréchet subdifferential at the projection point. More precisely, we have: 
Proposition 14 If $0<\varepsilon<\frac{1}{2 a}$, then, for every $x \in \mathbb{R}^{m}$, there exists $x_{\varepsilon} \in \operatorname{Dom}(\varphi)$ such that

$$
\frac{1}{2 \varepsilon}\left|x-x_{\varepsilon}\right|^{2}+\varphi\left(x_{\varepsilon}\right)=\varphi_{\varepsilon}(x) .
$$

Moreover, the following assertions hold.

(i) $J_{\varepsilon}(x):=x_{\varepsilon} \in \operatorname{Dom}\left(\partial^{-} \varphi\right)$ and $A_{\varepsilon}(x):=\frac{1}{\varepsilon}\left(x-x_{\varepsilon}\right) \in \partial^{-} \varphi\left(x_{\varepsilon}\right)$.

(ii) For all $x \in \mathbb{R}^{m}, x_{0} \in \operatorname{Dom}(\varphi)$ and all $0<\varepsilon<\frac{1}{4 a+1}$, the following inequality holds true

$$
\left|J_{\varepsilon}(x)-x\right|^{2} \leq \frac{1}{1-\varepsilon(4 a+1)}\left|x-x_{0}\right|^{2}+\frac{4 \varepsilon}{1-\varepsilon(4 a+1)}\left[\beta(|x|)+b^{2}+\varphi\left(x_{0}\right)\right],
$$

where $\beta(r)=a r^{2}+b r+c$. In particular, $J_{\varepsilon}$ and $A_{\varepsilon}$ are globally sublinear functions for $0<\varepsilon \leq$ $1 /(4 a+2)$, i.e.

$$
\left|J_{\varepsilon}(x)\right| \leq C(1+|x|), \quad\left|A_{\varepsilon}(x)\right| \leq \frac{C}{\varepsilon}(1+|x|), \quad \forall x \in \mathbb{R}^{m}, \text { where } C=C\left(a, b, c, x_{0}\right) .
$$

Moreover, if $x \in \bar{B}\left(x_{0}, r_{0}\right), r_{0}>0$, then

$$
\left|J_{\varepsilon}(x)-x\right| \leq\left(r_{0}+\sqrt{\varepsilon} C_{0}\right)(1-\varepsilon(4 a+1))^{-1 / 2},
$$

where $C_{0}=2 \sqrt{\beta\left(r_{0}+\left|x_{0}\right|\right)+b^{2}+\varphi\left(x_{0}\right)}$. Also, taking $x=x_{0}$ in (17), we get

$$
\lim _{\varepsilon \rightarrow 0} J_{\varepsilon}\left(x_{0}\right)=x_{0}, \forall x_{0} \in \operatorname{Dom}(\varphi) \quad \text { and } \quad \overline{\operatorname{Dom}\left(\partial^{-} \varphi\right)}=\operatorname{Dom}(\varphi)=\overline{\operatorname{Dom}(\varphi)} .
$$

(iii) In addition to its lower semicontinuity property, assume $\varphi$ to be $(\rho, \beta)$-semiconvex. We fix $x_{0} \in \operatorname{Dom}(\varphi)$ and $\lambda_{0}>0$. If we consider $0<r_{0} \leq \bar{r}_{0}$ and $0<\varepsilon \leq \bar{\varepsilon}_{0}$, with

$$
\bar{r}_{0}:=\frac{1}{36}\left(\frac{\lambda_{0}}{1+\lambda_{0}}\right)^{2} \frac{1}{\left(1+(\rho+\beta) \lambda_{0}\right)^{2}} \quad \text { and } \quad \bar{\varepsilon}_{0}:=\frac{1}{4 a+2} \wedge \frac{1-r_{0}}{4 a+1} \wedge \sqrt{r_{0}} \wedge \frac{r_{0}^{2}}{1+C_{0}^{2}}
$$

where $C_{0}=2 \sqrt{\beta\left(r_{0}+\left|x_{0}\right|\right)+b^{2}+\varphi\left(x_{0}\right)}$, then, for all $x, y \in \bar{B}\left(x_{0}, r_{0}\right)$, it follows

$$
\left|J_{\varepsilon}(x)-J_{\varepsilon}(y)\right| \leq\left(1+(\rho+\beta) \lambda_{0}\right)|x-y| \quad \text { and } \quad\left|A_{\varepsilon}(x)-A_{\varepsilon}(y)\right| \leq \frac{2+(\rho+\beta) \lambda_{0}}{\varepsilon}|x-y| .
$$

In particular, the minimizing point $J_{\varepsilon}(x)\left(=x_{\varepsilon}\right)$ of $\inf _{y \in \mathbb{R}^{m}}\left\{\frac{1}{2 \varepsilon}|x-y|^{2}+\varphi(y)\right\}$ is unique for $0<\varepsilon \leq \bar{\varepsilon}_{0}$ and $x \in \overline{\mathbb{B}}\left(\operatorname{Dom}(\varphi), \bar{r}_{0}\right)$. Moreover, $\varphi^{\varepsilon} \in C^{1}\left(\overline{\mathbb{B}}\left(\operatorname{Dom}(\varphi), \bar{r}_{0}\right)\right)$ and $\nabla \varphi^{\varepsilon}(x)=$ $A_{\varepsilon}(x) \in \partial^{-} \varphi\left(J_{\varepsilon} x\right)$. Moreover, $\nabla \varphi^{\varepsilon}$ and $J_{\varepsilon}$ are Lipschitz functions on every bounded subset of $\overline{\mathbb{B}}\left(\operatorname{Dom}(\varphi), \bar{r}_{0}\right)$ and $\operatorname{int}(\operatorname{Dom}(\varphi))=\operatorname{int}\left(\operatorname{Dom}\left(\partial^{-} \varphi\right)\right)$.

Proof. The interested reader can consult the complete proof of these technical results in [41, Proposition 4].

We are now able to give the main steps in the proof of Theorem 10 by hinting the main differences with respect to [41, Theorem 7]. Besides providing the reader with key elements, the proof is important for the developments on occupation measures. It is based on a penalization approach for the multivalued operator $\partial^{-} \varphi_{\mathcal{O}}$. However, since $\nabla \varphi_{\mathcal{O}}^{\varepsilon}$ is not defined on the entire space we must use a specific technique for this nonconvex setup, technique which is imposed also by the presence of the perturbing matrix $H$. For more details, the interested reader can consult [41, Theorem 7, Theorem 8].

Proof of Theorem 10. Let us fix $\eta \in \mathcal{O}=\operatorname{Dom}(\varphi)$. We recall that the positive quantities $\bar{\varepsilon}_{0}$ and $\bar{r}_{0}$ have been introduced in Proposition 14. 
Step 0. Approximating equations. We fix $0<r_{0}<\frac{\bar{r}_{0}}{4}$. One fixes a Lipschitz function $\alpha: \mathbb{R}^{m} \rightarrow$ $[0,1]$ such that

$$
\alpha(x)= \begin{cases}1, & \text { if } \quad d_{\mathcal{O}}(x) \leq \frac{\bar{r}_{0}}{3} \\ 0, & \text { if } \quad d_{\mathcal{O}}(x)>\frac{\bar{r}_{0}}{2}\end{cases}
$$

Next, one introduces the approximating equations, holding for Leb-almost all $t \in[0, T]$.

$$
y_{\varepsilon}(t)+\int_{t}^{T} H\left(r, y_{\varepsilon}(r)\right) \alpha\left(y_{\varepsilon}(r)\right) \nabla \varphi_{\mathcal{O}}^{\varepsilon}\left(y_{\varepsilon}(r)\right) d r=\eta+\int_{t}^{T} \int_{E} \alpha\left(y_{\varepsilon}(r)\right) \bar{f}\left(r, \gamma^{\prime}, y_{\varepsilon}(r)\right) \nu\left(d r, d \gamma^{\prime}\right) .
$$

The presence of the truncation $\alpha$ makes the integrand of the first integral correctly defined on the entire space (the reader may want to take a look at Proposition 14). It is clear that the set $\overline{\mathbb{B}}\left(\mathcal{O}, \bar{r}_{0} / 2\right)$ is invariant with respect to $(20)$. One may also want to note that the integrand function $\Psi_{\varepsilon}\left(r, y_{\varepsilon}(r)\right):=H\left(r, y_{\varepsilon}(r)\right) \alpha\left(y_{\varepsilon}(r)\right) \nabla \varphi_{\mathcal{O}}^{\varepsilon}\left(y_{\varepsilon}(r)\right)$ is represented as

$$
\Psi_{\varepsilon}\left(r, y_{\varepsilon}(r)\right)= \begin{cases}H\left(r, y_{\varepsilon}(r)\right) \nabla \varphi_{\mathcal{O}}^{\varepsilon}\left(y_{\varepsilon}(r)\right), & y_{\varepsilon}(r) \in \overline{\mathbb{B}}\left(\mathcal{O}, \frac{\bar{r}_{0}}{3}\right), \\ H\left(r, y_{\varepsilon}(r)\right) \alpha\left(y_{\varepsilon}(r)\right) \nabla \varphi_{\mathcal{O}}^{\varepsilon}\left(y_{\varepsilon}(r)\right), & y_{\varepsilon}(r) \in \overline{\mathbb{B}}\left(\mathcal{O}, \frac{\bar{r}_{0}}{2}\right) \backslash \overline{\mathbb{B}}\left(\mathcal{O}, \frac{\bar{r}_{0}}{3}\right), \\ 0, & y_{\varepsilon}(r) \notin \overline{\mathbb{B}}\left(\mathcal{O}, \frac{\bar{r}_{0}}{2}\right) .\end{cases}
$$

We emphasize that, in [41, Theorem 7], without the boundedness and closure of the domain, the authors provided first a local solution. Then, by adding the additional hypothesis on the domain, it was extended to a global solution. In our context, one can directly deduce that we have a global solution $y_{\varepsilon}$ for Eq.(20), solution which belongs to $C\left([0, T] ; \overline{\mathbb{B}}\left(\mathcal{O}, \bar{r}_{0}\right)\right)$.

Step 1. Estimates. The a priori estimates obtained, for the reversed time, in [41, Theorem 7 , Estimates (28)] lead to:

$$
\left\{\begin{array}{l}
\sup _{t \in[0 T]} \varphi_{\mathcal{O}}^{\varepsilon}\left(y_{\varepsilon}(t)\right)+\int_{0}^{T}\left|\nabla \varphi_{\mathcal{O}}^{\varepsilon}\left(y_{\varepsilon}(s)\right)\right|^{2} d s \leq C\left(r_{0}\right), \\
\int_{0}^{T}\left|y_{\varepsilon}(s)-J_{\varepsilon}\left(y_{\varepsilon}(s)\right)\right|^{2} d s \leq C\left(r_{0}\right) \varepsilon \quad \text { and } \quad \sup _{t \in[0, T]}\left|\nabla \varphi_{\mathcal{O}}^{\varepsilon}\left(y_{\varepsilon}(t)\right)\right| \leq \frac{2 C\left(r_{0}\right)}{\sqrt{\varepsilon}},
\end{array}\right.
$$

where $C\left(r_{0}\right)$ is a positive constant, independent of $\varepsilon$. The cited result in [41, Theorem 7] relies on local estimates (in particular $C$ is a local constant) but, in our framework, we use $\left(\mathrm{A}_{O}\right)$ to get a global constant $C\left(r_{0}\right)$.

Step 2. Limit equation. As usual for these approximating techniques, the next step consists in proving Cauchy behavior of the family of solutions and use the topological properties of the space $C\left([0, T] ; \overline{\mathbb{B}}\left(\mathcal{O}, \bar{r}_{0}\right)\right)$. Under the assumption of absolute continuity w.r.t the Lebesgue measure, one disintegrates the measure $\nu\left(d t, d \gamma^{\prime}\right)=\nu_{t}\left(d \gamma^{\prime}\right) d t$ and sets

$$
g(t, y):=\int_{E} \bar{f}\left(t, \gamma^{\prime}, y\right) \nu_{t}\left(d \gamma^{\prime}\right)
$$

for all $t \in[0, T]$ and all $y \in \mathbb{R}^{M}$. Next, one uses the chain differentiation rule for the (absolutely continuous) function

$$
\Phi_{\varepsilon, \delta}(r)=\left\langle M_{\varepsilon, \delta}(r)\left(y_{\varepsilon}(r)-y_{\delta}(r)\right), y_{\varepsilon}(r)-y_{\delta}(r)\right\rangle=\left|M_{\varepsilon, \delta}^{1 / 2}(r)\left(y_{\varepsilon}(r)-y_{\delta}(r)\right)\right|^{2},
$$

where, for $0<\varepsilon, \delta<\bar{\varepsilon}_{0}, M_{\varepsilon, \delta}(r):=\left[H\left(r, y_{\varepsilon}(r)\right)\right]^{-1}+\left[H\left(r, y_{\delta}(r)\right)\right]^{-1}$. We obtain, for every $0 \leq s \leq t \leq T$,

$$
\begin{aligned}
\Phi_{\varepsilon, \delta}(t) & =\Phi_{\varepsilon, \delta}(s)+\int_{s}^{t}\left[\left\langle\left(\alpha_{\varepsilon, \delta}(r) d y_{\varepsilon}(r)+\hat{\alpha}_{\varepsilon, \delta}(r) d y_{\delta}(r)\right)\left(y_{\varepsilon}(r)-y_{\delta}(r)\right), y_{\varepsilon}(r)-y_{\delta}(r)\right\rangle\right. \\
& \left.+2\left\langle M_{\varepsilon, \delta}(r)\left(y_{\varepsilon}(r)-y_{\delta}(r)\right),-H\left(r, y_{\varepsilon}(r)\right) \nabla \varphi_{\mathcal{O}}^{\varepsilon}\left(y_{\varepsilon}(r)\right)+H\left(r, y_{\delta}(r)\right) \nabla \varphi_{\mathcal{O}}^{\delta}\left(y_{\delta}(r)\right)\right\rangle\right] d r \\
& +2 \int_{s}^{t}\left\langle M_{\varepsilon, \delta}(r)\left(y_{\varepsilon}(r)-y_{\delta}(r)\right), \int_{E}\left(\bar{f}\left(r, \gamma^{\prime}, y_{\varepsilon}(r)\right)-\bar{f}\left(r, \gamma^{\prime}, y_{\delta}(r)\right)\right) \nu_{r}\left(d \gamma^{\prime}\right)\right\rangle d r
\end{aligned}
$$


with $\alpha_{\varepsilon, \delta}, \hat{\alpha}_{\varepsilon, \delta}: \mathbb{R}_{+} \rightarrow \mathcal{L}\left(\mathbb{R}^{m} ; \mathbb{R}^{m \times m}\right)$ two measurable functions, bounded by a constant independent of the processes $y_{\varepsilon}$ and $y_{\delta}$. Arguing similar to [41, Theorem 7, Page 95] and recalling that $\bar{f}$ is bounded, we deduce that

$$
\begin{aligned}
& \Phi_{\varepsilon, \delta}(t) \leq \Phi_{\varepsilon, \delta}(s)+C \int_{s}^{t}\left|y_{\varepsilon}-y_{\delta}\right|^{2}\left(C+\left|H\left(y_{\varepsilon}\right)\right|\left|\nabla \varphi_{\mathcal{O}}^{\varepsilon}\left(y_{\varepsilon}\right)\right|+\left|H\left(y_{\delta}\right)\right|\left|\nabla \varphi_{\mathcal{O}}^{\delta}\left(y_{\delta}\right)\right|\right) d r \\
& +2 \int_{s}^{t}\left\langle y_{\varepsilon}-y_{\delta},\left(\left[H\left(y_{\varepsilon}\right)\right]^{-1}-\left[H\left(y_{\delta}\right)\right]^{-1}\right)\left[H\left(y_{\varepsilon}\right) \nabla \varphi_{\mathcal{O}}^{\varepsilon}\left(y_{\varepsilon}\right)+H\left(y_{\delta}\right) \nabla \varphi_{\mathcal{O}}^{\delta}\left(y_{\delta}\right)\right]\right\rangle d r \\
& +4 \int_{s}^{t}\left(3\left|y_{\varepsilon}-y_{\delta}\right|^{2}+3 \varepsilon\left|\nabla \varphi_{\mathcal{O}}^{\varepsilon}\left(y_{\varepsilon}\right)\right|^{2}+3 \delta\left|\nabla \varphi_{\mathcal{O}}^{\delta}\left(y_{\delta}\right)\right|^{2}\right)\left(2 \rho+\beta\left|\nabla \varphi_{\mathcal{O}}^{\varepsilon}\left(y_{\varepsilon}\right)\right|+\beta\left|\nabla \varphi_{\mathcal{O}}^{\delta}\left(y_{\delta}\right)\right|\right) d r \\
& +4 \int_{s}^{t}\left(\varepsilon\left|\nabla \varphi_{\mathcal{O}}^{\varepsilon}\left(y_{\varepsilon}\right)\right|^{2}+\delta\left|\nabla \varphi_{\mathcal{O}}^{\delta}\left(y_{\delta}\right)\right|^{2}+(\varepsilon+\delta)\left\langle\nabla \varphi_{\mathcal{O}}^{\varepsilon}\left(y_{\varepsilon}\right), \nabla \varphi_{\mathcal{O}}^{\delta}\left(y_{\delta}\right)\right\rangle\right) d r \\
& +C \int_{s}^{t}\left(\left[H\left(y_{\varepsilon}\right)\right]^{-1}+\left[H\left(y_{\delta}\right)\right]^{-1}\right)\left|y_{\varepsilon}-y_{\delta}\right|^{2} d r .
\end{aligned}
$$

The reader may want to note that we have dropped the dependence on $r$ in the solutions $y$ and in $H$. These terms should, obviously, be read $y_{\varepsilon}(r), H\left(r, y_{\varepsilon}(r)\right)$, etc. To conclude, one simply plugs in the estimates (21) in order to get

$$
\begin{aligned}
& \text { - } \varepsilon \int_{s}^{t}\left|\nabla \varphi_{\mathcal{O}}^{\varepsilon}\left(y_{\varepsilon}(r)\right)\right|^{3} d r \leq \varepsilon \sup _{\tau \in[s, t]}\left|\nabla \varphi_{\mathcal{O}}^{\varepsilon}\left(y_{\varepsilon}(\tau)\right)\right| \int_{s}^{t}\left|\nabla \varphi_{\mathcal{O}}^{\varepsilon}\left(y_{\varepsilon}(r)\right)\right|^{2} d r \leq \sqrt{\varepsilon} C\left(r_{0}\right), \\
& \text { - } \varepsilon \int_{s}^{t}\left|\nabla \varphi_{\mathcal{O}}^{\varepsilon}\left(y_{\varepsilon}(r)\right)\right|^{2}\left|\nabla \varphi_{\mathcal{O}}^{\delta}\left(y_{\delta}(r)\right)\right| d r=2 \sqrt{\varepsilon} C\left(r_{0}\right) \int_{s}^{t}\left|\nabla \varphi_{\mathcal{O}}^{\varepsilon}\left(y_{\varepsilon}(r)\right)\right|\left|\nabla \varphi_{\mathcal{O}}^{\delta}\left(y_{\delta}(r)\right)\right| d r \\
& \quad \leq \sqrt{\varepsilon} C\left(r_{0}\right)\left(\int_{s}^{t}\left|\nabla \varphi_{\mathcal{O}}^{\varepsilon}\left(y_{\varepsilon}(r)\right)\right|^{2} d r\right)^{1 / 2}\left(\int_{s}^{t}\left|\nabla \varphi_{\mathcal{O}}^{\delta}\left(y_{\delta}(r)\right)\right|^{2} d r\right)^{1 / 2} \leq \sqrt{\varepsilon} C\left(r_{0}\right) .
\end{aligned}
$$

Finally, by passing to limit as $\varepsilon \rightarrow 0$, we deduce the existence of a pair $(y, k)$, which is a solution for Eq.(14) on $[0, T]$. The uniqueness follows patterns similar to these estimates and is omitted.

Remark 15 A careful look at [41, Theorem 7 Eq. (24-26, 28, 29)] shows that the constant $C\left(r_{0}\right)$ only depends on $r_{0}, c_{H}, \sup _{x \in \mathcal{O}}\left|\varphi_{\mathcal{O}}(x)\right|$, the bound and the Lipschitz constant of $\bar{f}$ (but not on $\bar{f}$ itself!) and T. Therefore, it takes the form $K\left(1+r_{0}+c_{H}+\sup _{x \in \mathcal{O}}\left|\varphi_{\mathcal{O}}(x)\right|+\sup _{x}|f(x)|+\sup _{x \neq y} \frac{|f(x)-f(y)|}{|x-y|}+T\right)$.

\subsection{Proofs of the Results of Section 4}

We give the proof of the linear formulations associated to the $\varepsilon$-approximating problems.

Proof of Proposition 11. We begin with proving that this set is non-empty. For simplicity reasons, we assume that the domain $\mathcal{O}$ is switch-invariant (i.e. $\mathcal{O}$ does not depend on $\gamma \in E$ ). The general result follows similar patterns and relies on the solution of the approximating problem

$$
\left\{\begin{array}{l}
-d Y_{t}^{\varepsilon, T, \xi, u}+H\left(t, Y_{t}^{\varepsilon, T, \xi, u}\right) \nabla \varphi_{\mathcal{O}_{\Gamma_{t-}}^{\varepsilon}}\left(Y_{t}^{\varepsilon, T, \xi, u}\right) d t \\
\quad=\int_{E} f\left(t, \gamma^{\prime}, \Gamma_{t-}, Y_{t-}^{\varepsilon, T, \xi, u}, Z_{t}^{\varepsilon, T, \xi, u}(\gamma), u_{t}\right) \widehat{q}\left(d t, d \gamma^{\prime}\right)-\int_{E} Z_{t}^{\varepsilon, T, \xi, u}\left(\gamma^{\prime}\right) q\left(d t, d \gamma^{\prime}\right), \\
Y_{T}^{\varepsilon, T, \xi, u}=\xi \in \mathbb{L}^{0}\left(\Omega, \mathcal{F}_{T}, \mathbb{P}^{\gamma_{0}} ; \mathbb{R}^{m}\right) .
\end{array}\right.
$$

Under this switch-invariance of the domain assumption, $\nabla \varphi_{\mathcal{O}}^{\varepsilon}$ is Lipschitz on $\overline{\mathbb{B}}\left(\mathcal{O}, \bar{r}_{0}\right)$ and we assume $\xi \in \mathbb{L}^{0}\left(\Omega, \mathcal{F}_{T}, \mathbb{P}^{\gamma_{0}} ; \mathcal{O}\right)$. The equation can be considered on the entire space by multiplying $H$ and $f$ with the function $\alpha$ appearing in Step 0 of the proof of Theorem 10. Then, one applies 
[16] to get the existence and uniqueness of the solution to (22). Moreover, due to [16], the càdlàg adapted process $Y^{\varepsilon, T, \xi, u}=\left(y^{\varepsilon, n}\right)$ satisfies

(23)

$$
\left\{\begin{array}{l}
y^{\varepsilon, M}\left(e_{M}(\omega), t\right)=\xi^{M}\left(e_{M}(\omega)\right) \text { and, for all } n<M, \\
-d y^{\varepsilon, n}\left(e_{n}(\omega), t\right)+H\left(t, y^{n}\left(e_{n}(\omega), t\right)\right) \nabla \varphi_{\mathcal{O}}^{\varepsilon}\left(y^{\varepsilon, n}\left(e_{n}(\omega), t\right)\right) d t \\
\quad=\sum_{\gamma \in E} f\left(\widehat{y}^{\varepsilon, n+1}\right)^{n}\left(e_{n}(\omega), s, \gamma^{\prime}, y^{\varepsilon, n}\left(e_{n}(\omega), s\right),-y^{\varepsilon, n}\left(e_{n}(\omega), s\right), u^{n}\left(e_{n}(\omega), s\right)\right) \widehat{q}_{e_{n}(\omega)}^{n}\left(d s,\left\{\gamma^{\prime}\right\}\right), \\
y^{\varepsilon, n}\left(e_{n}(\omega), T\right)=\xi^{n}\left(e_{n}(\omega)\right) .
\end{array}\right.
$$

In particular, $y^{\varepsilon, M} \in \overline{\mathbb{B}}\left(\mathcal{O}, \bar{r}_{0}\right) \subset \overline{\mathbb{B}}\left(0, C\left(r_{0}\right)\right)$ and the estimates (21) hold true. Moreover, the process $Z^{\varepsilon, T, \xi, u}=\left(z^{\varepsilon, n}\right)$ is given (as in (15)) by

$$
z^{\varepsilon, n}(e, t, \gamma):=y^{\varepsilon, n+1}(e \oplus(t, \gamma), t) 1_{t>|e|}-y^{\varepsilon, n}(e) .
$$

Hence, $z^{\varepsilon, n}$ is $\overline{\mathbb{B}}\left(0,2 C\left(r_{0}\right)\right)$-valued. (In the general case, $y^{\varepsilon, n}(e)$ might be replaced by $\Delta$ such that $z^{\varepsilon, n}$ is $\overline{\mathbb{B}}\left(0,2 C\left(r_{0}\right)+|\Delta|\right)$-valued $)$. One defines the occupation measure

$$
\mu_{\varepsilon}^{u(\cdot)} \in \mathcal{P}\left(\overline{\mathbb{B}}\left(0, C\left(r_{0}\right)\right)\right) \times \mathcal{P}\left([t, T] \times E^{2} \times \overline{\mathbb{B}}\left(0, C\left(r_{0}\right)\right) \times \mathbb{R}^{m} \times \overline{\mathbb{B}}\left(0,2 C\left(r_{0}\right)\right) \times U\right)
$$

by setting

$$
\begin{aligned}
& \mu_{\varepsilon}^{u(\cdot), 1}(A)=\mathbb{E}^{\gamma_{0}}\left[1_{A}\left(Y_{t}^{\varepsilon, T, \xi, u}\right)\right], \\
& \mu_{\varepsilon}^{u(\cdot), 2}(B)=\mathbb{E}^{\gamma_{0}}\left[\int_{t}^{T} 1_{B}\left(s, \gamma^{\prime}, \Gamma_{s-}, Y_{s-}^{\varepsilon, T, \xi, u}, \nabla \varphi_{\mathcal{O}}^{\varepsilon}\left(Y_{s}^{\varepsilon, T, \xi, u}\right), Z_{s}^{\varepsilon, T, \xi, u}\left(\gamma^{\prime}\right), u_{s}\right) d s\right],
\end{aligned}
$$

for all Borel sets $A \subset \overline{\mathbb{B}}\left(0, C\left(r_{0}\right)\right)$ and $B \subset[t, T] \times E^{2} \times \overline{\mathbb{B}}\left(0, C\left(r_{0}\right)\right) \times \mathbb{R}^{m} \times \overline{\mathbb{B}}\left(0,2 C\left(r_{0}\right)\right) \times U$. Due to Definition 4 and Proposition $14-(i i i)$, by noting that $\left(y_{1}, y_{2}\right)$ stands for $\left(Y^{\varepsilon, T, \xi, u}, \nabla \varphi_{\mathcal{O}}^{\varepsilon}\left(Y^{\varepsilon, T, \xi, u}\right)\right.$, one gets

$$
\begin{aligned}
& \operatorname{Supp}\left(\mu_{\varepsilon}^{u(\cdot), 2}\right) \subset \\
& \left\{\left(s, \gamma^{\prime}, \gamma, y_{1}, y_{2}, z, u\right): \forall p \in \mathbb{R}^{m},\left\langle p-y_{1}, y_{2}\right\rangle+\varphi_{\mathcal{O}}^{\varepsilon}\left(y_{1}\right) \leq \varphi_{\mathcal{O}_{\gamma}}^{\varepsilon}(p)+\left(\rho+\beta\left|y_{2}\right|\right)\left|p-y_{1}\right|^{2}\right\} .
\end{aligned}
$$

Moreover, the estimates (21) imply

$$
\begin{aligned}
& \int_{E^{\Delta, t, T} \times U} d_{\mathcal{O}_{\gamma}}^{2}\left(y_{1}\right) \mu_{\varepsilon}^{u(\cdot), 2}\left(d s d \gamma^{\prime} d \gamma d y_{1} d y_{2} d z d u\right) \leq \sup _{n} \int_{0}^{T}\left|y^{\varepsilon, n}(s)-J_{\varepsilon}\left(y^{\varepsilon, n}(s)\right)\right|^{2} d s \leq C\left(r_{0}\right) \varepsilon \\
& \int_{E^{\Delta, t, T} \times U}\left|y_{2}\right|^{2} \mu_{\varepsilon}^{u(\cdot), 2}\left(d s d \gamma^{\prime} d \gamma d y_{1} d y_{2} d z d u\right) \leq \sup _{n} \int_{0}^{T}\left|\nabla \varphi_{\mathcal{O}}^{\varepsilon}\left(y^{\varepsilon, n}(s)\right)\right|^{2} d s \leq C\left(r_{0}\right) .
\end{aligned}
$$

Finally, whenever $\phi \in C_{b}^{1,2}\left([t, T] \times \mathbb{R}^{m}\right)$, Itô's formula applied to $\phi\left(\cdot, Y^{\varepsilon, T, \xi, u}\right)$ on $[t, T]$ yields

$$
\begin{aligned}
& \mathbb{E}^{\gamma_{0}}[\phi(T, \xi)] \\
& =\mathbb{E}^{\gamma_{0}}\left[\phi\left(t, Y_{t}^{\varepsilon, T, \xi, u}\right)\right]+\mathbb{E}^{\gamma_{0}}\left[\int_{t}^{T}\left\langle\nabla_{y} \phi\left(s, Y_{s}^{\varepsilon, T, \xi, u}\right), H\left(s, Y_{s}^{\varepsilon, T, \xi, u}\right) \nabla \varphi_{\mathcal{O}}^{\varepsilon}\left(\left(Y_{s}^{\varepsilon, T, \xi, u}\right)\right\rangle d s\right]\right. \\
& -\mathbb{E}^{\gamma_{0}}\left[\int_{t}^{T}\left\langle\nabla_{y} \phi\left(s, Y_{s}^{\varepsilon, T, \xi, u}\right), \int_{E} f\left(s,, \gamma^{\prime}, \Gamma_{s-}, Y_{s-}^{\varepsilon, T, \xi, u}, Z_{s}^{\varepsilon, T, \xi, u}\left(\gamma^{\prime}\right), u_{s}\right) \lambda\left(\Gamma_{s-}\right) Q\left(\Gamma_{s-}, d \gamma^{\prime}\right)\right\rangle d s\right] \\
& +\mathbb{E}^{\gamma_{0}}\left[\int_{E} \phi\left(Y_{s-}^{\varepsilon, T, \xi, u}+Z_{s}^{\varepsilon, T, \xi, u}\left(\gamma^{\prime}\right)\right)-\phi\left(Y_{s-}^{\varepsilon, T, \xi, u}\right) \lambda\left(\Gamma_{s}\right) Q\left(\Gamma_{s}, \gamma^{\prime}\right) d s\right] .
\end{aligned}
$$

Using the definition of $\mu_{\varepsilon}^{u(\cdot)}$, one simply gets the linear constraint in $\Theta_{\varepsilon}(t, T, \xi)$. It follows that each occupation measure $\mu_{\varepsilon}^{u(\cdot)} \in \Theta_{\varepsilon}(t, T, \xi)$. 
The convexity of $\Theta_{\varepsilon}(t, T, \xi)$ is obvious.

Relative compactness w.r.t. the weak * topology of probability measures follows from the second inequality in (25) by noting that all the other components are bounded and simply applying Prohorov's theorem. Finally, let us consider some sequence $\Theta_{\varepsilon}(t, T, \xi) \ni \mu_{m} \rightarrow \mu$. We only need to prove the support condition. To this purpose, we let

$$
\mathcal{S}:=\left\{\left(s, \gamma^{\prime}, \gamma, y_{1}, y_{2}, z, u\right): \forall p \in \mathbb{R}^{m},\left\langle p-y_{1}, y_{2}\right\rangle+\varphi_{\mathcal{O}}^{\varepsilon}\left(y_{1}\right) \leq \varphi_{\mathcal{O}}^{\varepsilon}(p)+\left(\rho+\beta\left|y_{2}\right|\right)\left|p-y_{1}\right|^{2}\right\} .
$$

This set is closed. Thus, $0=\lim _{n} \inf \mu_{m}^{2}\left(\mathcal{S}^{c}\right) \geq \mu\left(\mathcal{S}^{c}\right)$. This completes our proof.

To end this section, we give the proof of Theorem 12 characterizing the (relaxed) occupation measures associated to the BSVI (1). Before giving the proof of this theorem, we invite the reader to note that if one passes to the limit as $\varepsilon \rightarrow 0$ in (23) and looks at the proof of Theorem 10, one gets a solution of (14). Then, one obtains the solution of (1). Therefore, the limits of the occupation measures introduced before characterize (but may not be limited to) all the controlled solutions of (1). This justifies our interest in the properties of such $\Theta_{0}(t, T, \xi)$.

Proof of Theorem 12. (i) Convexity and closedness follow immediately from the properties of the approximating sets $\Theta_{\varepsilon}(t, T, \xi)$. For details on limits of sets, the reader is referred to [2, Chapter 1, Section 1.1]. To see that this limit set is non-empty, one simply recalls that the second order moment inequalities in the definition of $\Theta_{\varepsilon}(t, T, \xi)$ are uniform w.r.t. $\varepsilon>0$. Then, one uses Prohorov's theorem (see, for example [7]). Finally, since the application $E^{\Delta, t, T} \times U \ni\left(s, \gamma^{\prime}, \gamma, y_{1}, y_{2}, z, u\right) \mapsto$ $\left|y_{2}\right|^{2}$ is weakly lower semicontinuous, the moment estimate follows.

(ii) Whenever $\left(\mu^{\varepsilon} \in \Theta_{\varepsilon}(t, T, \xi)\right)_{\varepsilon>0}$ is a sequence converging to $\mu \in \Theta_{0}(t, T, \xi)$, one has

$$
\int_{E^{\Delta, t, T} \times U}\left(d_{\mathcal{O}_{\gamma}}^{2} \wedge 1\right)\left(y_{1}\right) \mu^{\varepsilon, 2}\left(d s d \gamma^{\prime} d \gamma d y_{1} d y_{2} d z d u\right) \leq C\left(r_{0}\right) \varepsilon,
$$

for all $\varepsilon>0$. Therefore, passing to the limit as $\varepsilon \rightarrow 0$ yields $y_{1} \in \mathcal{O}_{\gamma}, \mu^{2}$ - almost everywhere on $E^{\Delta, t, T} \times U$. To prove the second condition, we introduce the closed, convex sets

$$
\begin{aligned}
\mathcal{S}_{\varepsilon} & :=\left\{\left(s, \gamma^{\prime}, \gamma, y_{1}, y_{2}, z, u\right): \forall p \in \mathbb{R}^{m},\left\langle p-y_{1}, y_{2}\right\rangle+\varphi_{\mathcal{O}_{\gamma}}^{\varepsilon}\left(y_{1}\right) \leq \varphi_{\mathcal{O}_{\gamma}}(p)+\left(\rho+\beta\left|y_{2}\right|\right)\left|p-y_{1}\right|^{2}\right\}, \\
\mathcal{S} & :=\left\{\begin{array}{c}
\left(s, \gamma^{\prime}, \gamma, y_{1}, y_{2}, z, u\right): y_{1} \in \mathcal{O}_{\gamma}, \forall p \in \mathbb{R}^{m}, \\
\left\langle p-y_{1}, y_{2}\right\rangle+\varphi_{\mathcal{O}_{\gamma}}\left(y_{1}\right) \leq \varphi_{\mathcal{O}_{\gamma}}(p)+\left(\rho+\beta\left|y_{2}\right|\right)\left|p-y_{1}\right|^{2}
\end{array}\right\} .
\end{aligned}
$$

One recalls that $\operatorname{Supp}\left(\mu^{\varepsilon, 2}\right) \subset \mathcal{S}_{\varepsilon}$. Second, $\mathcal{S}_{\varepsilon}$ is increasing (as a set-valued function of $\varepsilon$ ) and

$$
\mu^{2}\left(\mathcal{S}^{c}\right)=\mu^{2}\left(\underset{\varepsilon>0}{\cup} \mathcal{S}_{\varepsilon}^{c}\right) \leq \liminf _{\varepsilon \rightarrow 0+} \mu^{\varepsilon, 2}\left(\mathcal{S}_{\varepsilon}^{c}\right)=0
$$

(iii) One simply notes that the sets appearing in the right-hand side of condition (iii) are convex and compact. This is a simple consequence of gradient inequalities in (21). The assertion follows by passing to the limit as $\varepsilon \rightarrow 0$ in the equality constraints characterizing the sets $\Theta_{\varepsilon}(t, T, \xi)$. Our proof is now complete.

\section{References}

[1] I. Asiminoaei and A. Rascanu. Approximation and simulation of stochastic variational inequalitie-splitting up method. Numer. Funct. Anal. Optim., 18(3-4):251-282, 1996.

[2] J.P. Aubin and H. Frankowska. Set-valued analysis. Birkhäuser, Boston, 1990.

[3] Vlad Bally and Victor Rabiet. Asymptotic behavior for multi-scale PDMP's. working paper or preprint, April 2015. 
[4] V. Barbu. Optimal control of variational inequalities, volume 100 of Research Notes in Mathematics. Pitman (Advanced Publishing Program), Boston, MA, 1984.

[5] Guy Barles, Rainer Buckdahn, and Etienne Pardoux. Backward stochastic differential equations and integral-partial differential equations. Stochastics and Stochastic Reports, 60(1-2):5783, 1997.

[6] A.G. Bhatt and V.S. Borkar. Occupation measures for controlled Markov processes: Characterization and optimality. Ann. of Probability, 24:1531-1562, 1996.

[7] Patrick Billingsley. Convergence of probability measures. Wiley Series in Probability and Statistics: Probability and Statistics. John Wiley \& Sons Inc., New York, second edition, 1999. A Wiley-Interscience Publication.

[8] Jean-Michel Bismut. Conjugate convex functions in optimal stochastic control. J. Math. Anal. Appl., 44:384-404, 1973.

[9] V. Borkar and V. Gaitsgory. Averaging of singularly perturbed controlled stochastic differential equations. Appl. Math. Optimization, 56(2):169-209, 2007.

[10] Pierre Brémaud. Point processes and queues : martingale dynamics. Springer series in statistics. Springer-Verlag, New York, 1981.

[11] H. Brézis. Opérateurs maximaux monotones et semi-groupes de contractions dans les espaces de Hilbert. North-Holland Publishing Co., Amsterdam-London; American Elsevier Publishing Co., Inc., New York, 1973. North-Holland Mathematics Studies, No. 5. Notas de Matemática (50).

[12] R. Buckdahn, D. Goreac, and M. Quincampoix. Stochastic optimal control and linear programming approach. Appl. Math. Optimization, 63(2):257-276, 2011.

[13] R. Carbone, B. Ferrario, and M. Santacroce. Backward stochastic differential equations driven by càdlàg martingales. Teor. Veroyatn. Primen., 52(2):375-385, 2007.

[14] Samuel N. Cohen and Robert J. Elliott. Comparisons for backward stochastic differential equations on Markov chains and related no-arbitrage conditions. Ann. Appl. Probab., 20(1):267-311, 2010 .

[15] Fulvia Confortola and Marco Fuhrman. Backward stochastic differential equations associated to jump Markov processes and applications. Stochastic Process. Appl., 124(1):289-316, 2014.

[16] Fulvia Confortola, Marco Fuhrman, and Jean Jacod. Backward stochastic differential equations driven by a marked point process: an elementary approach, with an application to optimal control. Annals of Applied Probability, to appear, 2015. arXiv:1407.0876.

[17] D. L. Cook, A. N. Gerber, and S. J. Tapscott. Modelling stochastic gene expression: Implications for haploinsufficiency. Proc. Natl. Acad. Sci. USA, 95:15641-15646, 1998.

[18] O. L. V. Costa and F. Dufour. A linear programming formulation for constrained discounted continuous control for piecewise deterministic Markov processes. J. Math. Anal. Appl., 424(2):892-914, 2015.

[19] A. Crudu, A. Debussche, A. Muller, and O. Radulescu. Convergence of stochastic gene networks to hybrid piecewise deterministic processes. The Annals of Applied Probability, 22(5):1822-1859, 102012. 
[20] A. Crudu, A. Debussche, and O. Radulescu. Hybrid stochastic simplifications for multiscale gene networks. BMC Systems Biology, page 3:89, 2009.

[21] M. H. A. Davis. Piecewise-deterministic Markov-processes - A general-class of non-diffusion stochastic-models. Journal of the Royal Statistical Society Series B-Methodological, 46(3):353388, 1984.

[22] M. H. A. Davis. Markov models and optimization, volume 49 of Monographs on Statistics and Applied Probability. Chapman \& Hall, London, 1993.

[23] Francois Dufour and Richard H. Stockbridge. On the existence of strict optimal controls for constrained, controlled Markov processes in continuous time. Stochastics, 84(1):55-78, 2012.

[24] V. Gaitsgory and M. Quincampoix. Linear programming approach to deterministic infinite horizon optimal control problems with discouting. SIAM J. Control Optimization, 48(4):2480 $2512,2009$.

[25] Anouar M. Gassous, Aurel Răşcanu, and Eduard Rotenstein. Multivalued backward stochastic differential equations with oblique subgradients. Stochastic Process. Appl., 125(8):3170-3195, 2015 .

[26] Daniel T. Gillespie. Exact stochastic simulation of coupled chemical reactions. The Journal of Physical Chemistry, 81(25):2340-2361, 1977.

[27] R. Goebel and R.T. Rockafellar. Generalized conjugacy in hamilton-jacobi theory for fully convex lagrangians. Journal of Convex Analysis, 9(2), 2002.

[28] Dan Goreac. Viability, Invariance and Reachability for Controlled Piecewise Deterministic Markov Processes Associated to Gene Networks. ESAIM-Control Optimisation and Calculus of Variations, 18(2):401-426, APR 2012.

[29] Dan Goreac and Oana-Silvia Serea. Linearization Techniques for Controlled Piecewise Deterministic Markov Processes; Application to Zubov's Method. Applied Mathematics and Optimization, 66:209-238, 2012.

[30] Dan Goreac and Oana-Silvia Serea. Optimality issues for a class of controlled singularly perturbed stochastic systems. Journal of Optimization Theory and Applications, 168(1):22$52,2015$.

[31] C.M. Guldberg and P. Waage. Studies Concerning Affinity. C. M. Forhandlinger: VidenskabsSelskabet i Christiana, 35, 1864.

[32] J. Hasty, J. Pradines, M. Dolnik, and J.J. Collins. Noise-based switches and amplifiers for gene expression. PNAS, 97(5):2075-2080, 2000.

[33] Ying Hu and Shanjian Tang. Multi-dimensional BSDE with oblique reflection and optimal switching. Probab. Theory Related Fields, 147(1-2):89-121, 2010.

[34] N. Ikeda and S. Watanabe. Stochastic Differential Equations and Diffusion Processes, volume 24 of North-Holland Mathematical Library. North-Holland Publishing Co., AmsterdamNew York; Kodansha, Ltd., Tokyo, 1981.

[35] Idris Kharroubi and Thomas Lim. Progressive enlargement of filtrations and backward stochastic differential equations with jumps. J. Theoret. Probab., 27(3):683-724, 2014.

[36] T.G. Kurtz and R.H. Stockbridge. Existence of Markov controls and characterization of optimal Markov control. SIAM J. Control Optim., 36(2):609-653, 1998. 
[37] J.B. Lasserre, D. Henrion, C. Prieur, and Trélat. E. Nonlinear optimal control via occupational measures and LMI-Relaxations. SIAM J. Control Optim., 47(4):1643-1666, 2008.

[38] E. Pardoux and S.G. Peng. Adapted solution of a backward stochastic differential equation. Syst. Control Lett., 14(1):55 - 61, 1990.

[39] Etienne Pardoux and Aurel Răşcanu. Backward stochastic differential equations with subdifferential operator and related variational inequalities. Stochastic Process. Appl., 76(2):191-215, 1998.

[40] Etienne Pardoux and Aurel Răşcanu. Backward stochastic variational inequalities. Stochastics Stochastics Rep., 67(3-4):159-167, 1999.

[41] Aurel Răşcanu and Eduard Rotenstein. A non-convex setup for multivalued differential equations driven by oblique subgradients. Nonlinear Anal., 111:82-104, 2014.

[42] R.H. Stockbridge. Time-average control of a martingale problem. Existence of a stationary solution. Ann. of Probability, 18:190-205, 1990.

[43] Shan Jian Tang and Xun Jing Li. Necessary conditions for optimal control of stochastic systems with random jumps. SIAM J. Control Optim., 32(5):1447-1475, 1994.

[44] Jianming Xia. Backward stochastic differential equation with random measures. Acta Math. Appl. Sinica (English Ser.), 16(3):225-234, 2000.

[45] G.G. Yin and Q. Zhang. Continuous- Time Markov Chains and Applications. A singular Perturbation Approach. Springer-Verlag, New York, 1997. 